\title{
On the classification of Darboux integrable chains
}

\author{
Ismagil Habibullin, ${ }^{\text {a) }}$ Natalya Zheltukhina, ${ }^{\text {b) }}$ and Aslı Pekcan \\ Department of Mathematics, Faculty of Science, Bilkent University, \\ 06800 Ankara, Turkey
}

(Received 25 June 2008; accepted 10 September 2008; published online 9 October 2008)

We study a differential-difference equation of the form $t_{x}(n+1)=f(t(n), t(n+1)$, $\left.t_{x}(n)\right)$ with unknown $t=t(n, x)$ depending on $x$ and $n$. The equation is called a Darboux integrable if there exist functions $F$ (called an $x$-integral) and $I$ (called an $n$-integral), both of a finite number of variables $x, t(n), t(n \pm 1), t(n \pm 2), \ldots$, $t_{x}(n), t_{x x}(n), \ldots$, such that $D_{x} F=0$ and $D I=I$, where $D_{x}$ is the operator of total differentiation with respect to $x$ and $D$ is the shift operator: $D p(n)=p(n+1)$. The Darboux integrability property is reformulated in terms of characteristic Lie algebras that give an effective tool for classification of integrable equations. The complete list of equations of the form above admitting nontrivial $x$-integrals is given in the case when the function $f$ is of the special form $f(x, y, z)=z+d(x, y)$. (C) 2008 American Institute of Physics. [DOI: 10.1063/1.2992950]

\section{INTRODUCTION}

In this paper we study integrable semidiscrete chains of the following form:

$$
t_{x}(n+1)=f\left(t(n), t(n+1), t_{x}(n)\right),
$$

where the unknown $t=t(n, x)$ is a function of two independent variables: discrete $n$ and continuous $x$. Chain (1) can also be interpreted as an infinite system of ordinary differential equations for the sequence of the variables $\{t(n)\}_{n=-\infty}^{\infty}$. Here $f=f\left(t, t_{1}, t_{x}\right)$ is assumed to be a locally analytical function of three variables satisfying at least locally the condition

$$
\frac{\partial f}{\partial t_{x}} \neq 0
$$

For the sake of convenience we introduce subindex denoting shifts $t_{k}=t(n+k, x)\left(\right.$ keep $\left.t_{0}=t\right)$ and derivatives $t_{x}=(\partial / \partial x) t(n, x), t_{x x}=\left(\partial^{2} / \partial x^{2}\right) t(n, x)$, and so on. We denote through $D$ and $D_{x}$ the shift operator and, correspondingly, the operator of total derivative with respect to $x$. For instance, $D h(n, x)=h(n+1, x)$ and $D_{x} h(n, x)=(\partial / \partial x) h(n, x)$. Set of all the variables $\left\{t_{k}\right\}_{k=-\infty}^{\infty} ;\left\{D_{x}^{m} t_{m=1}^{\infty}\right.$ constitutes the set of dynamical variables. Below we consider the dynamical variables as independent ones. Since in the literature the term "integrable" has various meanings let us specify the meaning used in the article. Introduce first notions of $n$ - and $x$-integrals. ${ }^{1}$

Functions $I$ and $F$, both depending on $x$ and a finite number of dynamical variables, are called, respectively, $n$ - and $x$-integrals of (1) if $D I=I$ and $D_{x} F=0$.

Definition: Chain (1) is called integrable (Darboux integrable) if it admits a nontrivial $n$-integral and a nontrivial $x$-integral.

Darboux integrability implies the so-called $C$-integrability. Knowing both integrals $F$ and $I$ a Cole-Hopf-type differential substitution $w=F+I$ reduces Eq. (1) to the discrete version of the D'Alembert wave equation, $w_{1 x}-w_{x}=0$. Indeed, $(D-1) D_{x}(w)=(D-1) D_{x} F+D_{x}(D-1) I=0$.

\footnotetext{
${ }^{a)}$ Electronic mail: habibullin_i@mail.rb.ru. On leave from Ufa Institute of Mathematics, Russian Academy of Science, Chernyshevskii Str., 112, Ufa 450077, Russia.

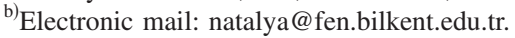


It is remarkable that an integrable chain is reduced to a pair consisting of an ordinary differential equation and an ordinary difference equation. To illustrate it, note first that any $n$-integral might depend only on $x$ - and $x$-derivatives of the variable $t, I=I\left(x, t, t_{x}, t_{x x}, \ldots\right)$, and similarly any $x$-integral depends only on $x$ and the shifts, $F=F\left(x, t, t_{ \pm 1}, t_{ \pm 2}, \ldots\right)$. Therefore each solution of integrable chain (1) satisfies two equations:

$$
I\left(x, t, t_{x}, t_{x x}, \ldots\right)=p(x), \quad F\left(x, t, t_{ \pm 1}, t_{ \pm 2}, \ldots\right)=q(n),
$$

with properly chosen functions $p(x)$ and $q(n)$.

Nowadays the discrete phenomena are studied intensively due to their various applications in physics. For the discussions and references we refer to the articles in Refs. 1-5.

Chain (1) is very close to a well studied object-the partial differential equation of the hyperbolic type

$$
u_{x y}=f\left(x, y, u, u_{x}, u_{y}\right) .
$$

The definition of integrability for Eq. (3) was introduced by Darboux. The famous Liouville equation $u_{x y}=e^{u}$ provides an illustrative example of the Darboux integrable equation. An effective criterion of integrability of (3) was discovered by Darboux himself: Eq. (3) is integrable if and only if the Laplace sequence of the linearized equation terminates at both ends (see Refs. 6-8). This criterion of integrability was used in Ref. 8 where the complete list of all Darboux integrable equations of form (3) is given.

An alternative approach to the classification problem based on the notion of the characteristic Lie algebra of hyperbolic-type systems was introduced years ago in Refs. 9 and 10. In these articles an algebraic criterion of Darboux integrability property has been formulated. An important classification result was obtained in Ref. 9 for the exponential system

$$
u_{x y}^{i}=\exp \left(a_{i 1} u^{1}+a_{i 2} u^{2}+\cdots+a_{i n} u^{n}\right), \quad i=1,2, \ldots, n .
$$

It was proved that system (4) is a Darboux integrable if and only if the matrix $A=\left(a_{i j}\right)$ is the Cartan matrix of a semisimple Lie algebra. Properties of the characteristic Lie algebras of the hyperbolic systems

$$
u_{x y}^{i}=c_{j k}^{i} u^{j} u^{k}, \quad i, j, k=1,2, \ldots, n,
$$

have been studied in Refs. 11 and 12. Hyperbolic systems of general form admitting integrals are studied in Ref. 13. A promising idea of adopting the characteristic Lie algebras to the problem of classification of the hyperbolic systems which are integrated by means of the inverse scattering transforms method is discussed in Ref. 14.

The method of characteristic Lie algebras is closely connected with the symmetry approach ${ }^{15}$ which is proved to be a very effective tool to classify integrable nonlinear equations of evolutionary type ${ }^{16-20}$ (see also the survey in Ref. 3 and references therein). However, the symmetry approach meets very serious difficulties when applied to hyperbolic-type models. After the papers in Refs. 21 and 22 it became clear that this case needs alternative methods.

In this article an algorithm of classification of integrable discrete chains of form (1) is suggested based on the notion of the characteristic Lie algebra (see also Refs. 23-25).

To introduce the characteristic Lie algebra $L_{n}$ of (1) in the direction of $n$, note that

$$
D^{-j} \frac{\partial}{\partial t_{1}} D^{j} I=0
$$

for any $n$-integral $I$ and $j \geq 1$. Indeed, the equation $D I=I$ can be rewritten in an enlarged form as

$$
I\left(x, n+1, t_{1}, f, f_{x}, f_{x x}, \ldots\right)=I\left(x, n, t, t_{x}, t_{x x}, \ldots\right) .
$$

The left hand side $D I$ of equality (7) contains the variable $t_{1}$, while the right hand side does not. Hence, $\left(\partial / \partial t_{1}\right)(D I)=0$, which implies 


$$
D^{-1} \frac{\partial}{\partial t_{1}} D I=0
$$

Proceeding this way one can easily prove (6) from the equality $D^{j} I=I, j \geq 1$.

Define vector fields

$$
Y_{j}=D^{-j} \frac{\partial}{\partial t_{1}} D^{j}, \quad j \geq 1
$$

and

$$
X_{j}=\frac{\partial}{\partial t_{-j}}, \quad j \geq 1 .
$$

We have $Y_{j} I=0$ and $X_{j} I=0$ for any $n$-integral $I$ of (1) and $j \geq 1$. The following theorem (see Ref. 24) defines the characteristic Lie algebra $L_{n}$ of (1).

Theorem 1: Equation (1) admits a nontrivial $n$-integral if and only if the following two conditions hold:

(1) Linear space spanned by the operators $\left\{Y_{j}\right\}_{1}^{\infty}$ is of finite dimension. We denote this dimension by $N$.

(2) Lie algebra $L_{n}$ generated by the operators $Y_{1}, Y_{2}, \ldots, Y_{N}, X_{1}, X_{2}, \ldots, X_{N}$ is of finite dimension. We call $L_{n}$ the characteristic Lie algebra of (1) in the direction of $n$.

To introduce the characteristic Lie algebra $L_{x}$ of (1) in the direction of $x$, note that Eq. (1) due to (2) can be rewritten as $t_{x}(n-1)=g\left(t(n), t(n-1), t_{x}(n)\right)$. An $x$-integral $F\left(x, t, t_{ \pm 1}, t_{ \pm 2}, \ldots\right)$ solves the equation $D_{x} F=0$, i.e., $K_{0} F=0$, where

$$
K_{0}=\frac{\partial}{\partial x}+t_{x} \frac{\partial}{\partial t}+f \frac{\partial}{\partial t_{1}}+g \frac{\partial}{\partial t_{-1}}+f_{1} \frac{\partial}{\partial t_{2}}+g_{-1} \frac{\partial}{\partial t_{-2}}+\cdots .
$$

Since $F$ does not depend on the variable $t_{x}$ one gets $X F=0$, where

$$
X=\frac{\partial}{\partial t_{x}} .
$$

Therefore, any vector field from the Lie algebra generated by $K_{0}$ and $X$ annulates $F$. This algebra is called the characteristic Lie algebra $L_{x}$ of chain (1) in the $x$-direction.

The following result is essential. Its proof is a simple consequence of the famous Jacobi theorem (the Jacobi theorem is discussed, for instance, in Ref. 10).

Theorem 2: Equation (1) admits a nontrivial $x$-integral if and only if its Lie algebra $L_{x}$ is of finite dimension.

In the present paper we restrict ourselves to consideration of the existence of $x$-integrals for a particular kind of chain (1), namely, we study chains of the form

$$
t_{1 x}=t_{x}+d\left(t, t_{1}\right)
$$

admitting nontrivial $x$-integrals. The main result of the paper, Theorem 3 below, is the complete list of chains (12) admitting nontrivial $x$-integrals.

Theorem 3: Chain (12) admits a nontrivial $x$-integral if and only if $d\left(t, t_{1}\right)$ is one of the following kinds:

(2) $d\left(t, t_{1}\right)=c_{0} t\left(t-t_{1}\right)+c_{2}\left(t-t_{1}\right)^{2}+c_{3} t-c_{3} t_{1}$,

(3) $d\left(t, t_{1}\right)=A\left(t-t_{1}\right) e^{\alpha t}$, 
(4) $d\left(t, t_{1}\right)=c_{4}\left(e^{\alpha t_{1}}-e^{\alpha t}\right)+c_{5}\left(e^{-\alpha t_{1}}-e^{-\alpha t}\right)$, where $A=A\left(t-t_{1}\right)$ is a function of $\tau=t-t_{1}$ and $c_{0}-c_{5}$ are some constants with $c_{0} \neq 0, c_{4} \neq 0$, and $c_{5} \neq 0$, and $\alpha$ is a nonzero constant. Moreover, $x$-integrals in each of the cases are

(i) $F=x+\int^{\tau} d u / A(u)$ if $A(u) \neq 0$ and $F=t_{1}-t$ if $A(u) \equiv 0$,

(ii) $\quad F=\left(1 /\left(-c_{2}-c_{0}\right)\right) \ln \left|\left(-c_{2}-c_{0}\right) \tau_{1} / \tau_{2}+c_{2}\right|+1 / c_{2} \ln \left|c_{2} \tau_{1} / \tau-c_{2}-c_{0}\right|$ for $c_{2}\left(c_{2}+c_{0}\right) \neq 0, F=\ln \tau_{1}$ $-\ln \tau_{2}+\tau_{1} / \tau$ for $c_{2}=0$, and $F=\tau_{1} / \tau_{2}-\ln \tau+\ln \tau_{1}$ for $c_{2}=-c_{0}$,

(iii) $F=\int^{\tau} e^{-\alpha u} d u / A(u)-\int^{\tau_{1}} d u / A(u)$, and

(iv) $F=\left[\left(e^{\alpha t}-e^{\alpha t_{2}}\right)\left(e^{\alpha t_{1}}-e^{\alpha t_{3}}\right)\right] /\left[\left(e^{\alpha t}-e^{\alpha t_{3}}\right)\left(e^{\alpha t_{1}}-e^{\alpha t_{2}}\right)\right]$.

The $n$-integrals of chain (12) can be studied in a similar way by using Theorem 1, but this problem is out of the frame of the present article.

The article is organized as follows. In Sec. II, by using the properly chosen sequence of multiple commutators, a very rough classification result is obtained: function $d\left(t, t_{1}\right)$ for chain (12) admitting $x$-integrals is a quasipolynomial on $t$ with coefficients depending on $\tau=t-t_{1}$. Then it is observed that the exponents $\alpha_{0}=0, \alpha_{1}, \ldots, \alpha_{s}$ in expansion (26) cannot be arbitrary. For example, if the coefficient before $e^{\alpha_{0} t}=1$ is not identically zero, then the quasipolynomial $d\left(t, t_{1}\right)$ is really a polynomial on $t$ with coefficients depending on $\tau$. In Sec. III we prove that the degree of this polynomial is at most 1. If $d$ contains a term of the form $\mu(\tau) t^{j} e^{\alpha_{k} t}$ with $\alpha_{k} \neq 0$, then $j=0$ (Sec. IV). In Sec. $\mathrm{V}$ it is proved that if $d$ contains terms with $e^{\alpha_{k} t}$ and $e^{\alpha_{j} t}$ having nonzero exponents, then $\alpha_{k}=-\alpha_{j}$. This last case contains chains having infinite dimensional characteristic Lie algebras for which the sequence of multiple commutators grows very slowly. They are studied in Secs. VI and VII. One can find the well known semidiscrete version of the sine-Gordon (SG) model among them. It is worth mentioning that in Sec. VII the characteristic Lie algebra $L_{x}$ for semidiscrete SG is completely described. The last section, Sec. VIII, contains the proof of the main theorem, Theorem 3, and here the method of constructing $x$-integrals is also briefly discussed.

\section{A NECESSARY INTEGRABILITY CONDITION}

Define a class $\mathbf{F}$ of locally analytical functions each of which depends only on a finite number of dynamical variables. In particular, we assume that $f\left(t, t_{1}, t_{x}\right) \in \mathbf{F}$. We will consider vector fields given as an infinite formal series of the form

$$
Y=\sum_{-\infty}^{\infty} y_{k} \frac{\partial}{\partial t_{k}},
$$

with coefficients $y_{k} \in \mathbf{F}$. Introduce notions of linearly dependent and independent sets of vector fields (13). Denote through $P_{N}$ the projection operator acting according to the rule

$$
P_{N}(Y)=\sum_{k=-N}^{N} y_{k} \frac{\partial}{\partial t_{k}} .
$$

First we consider finite vector fields as

$$
Z=\sum_{k=-N}^{N} z_{k} \frac{\partial}{\partial t_{k}} .
$$

We say that a set of finite vector fields $Z_{1}, Z_{2}, \ldots, Z_{m}$ is linearly dependent in some open region $\mathbf{U}$ if there is a set of functions $\lambda_{1}, \lambda_{2}, \ldots, \lambda_{m}$ defined on $\mathbf{U}$ such that the function $\left|\lambda_{1}\right|^{2}+\left|\lambda_{2}\right|^{2}+\cdots$ $+\left|\lambda_{m}\right|^{2}$ does not vanish identically and the condition

$$
\lambda_{1} Z_{1}+\lambda_{2} Z_{2}+\cdots+\lambda_{m} Z_{m}=0
$$

holds for each point of region $\mathbf{U}$. 
We call a set of the vector fields $Y_{1}, Y_{2}, \ldots, Y_{m}$ of form (13) linearly dependent in region $\mathbf{U}$ if for each natural $N$ the set of finite vector fields $P_{N}\left(Y_{1}\right), P_{N}\left(Y_{2}\right), \ldots, P_{N}\left(Y_{m}\right)$ is linearly dependent in this region. Otherwise we call the set $Y_{1}, Y_{2}, \ldots, Y_{m}$ linearly independent in $\mathbf{U}$.

The following proposition is very useful. Its proof is almost evident.

Proposition: If a vector field $Y$ is expressed as a linear combination,

$$
Y=\lambda_{1} Y_{1}+\lambda_{2} Y_{2}+\cdots+\lambda_{m} Y_{m},
$$

where the set of vector fields $Y_{1}, Y_{2}, \ldots, Y_{m}$ is linearly independent in $\boldsymbol{U}$ and the coefficients of all the vector fields $Y, Y_{1}, Y_{2}, \ldots, Y_{m}$ belonging to $\boldsymbol{F}$ are defined in $\boldsymbol{U}$, then the coefficients $\lambda_{1}, \lambda_{2}, \ldots, \lambda_{m}$ are in $\boldsymbol{F}$.

Below we concentrate on the class of chains of form (12). For this case the Lie algebra $L_{x}$ splits down into a direct sum of two subalgebras. Indeed, since $f=t_{x}+d$ and $g=t_{x}-d_{-1}$ one gets $f_{k}=t_{x}+d+\sum_{j=1}^{k} d_{j}$ and $g_{-k}=t_{x}-\sum_{j=1}^{k+1} d_{-k}$ for $k \geq 1$, where $d=d\left(t, t_{1}\right)$ and $d_{j}=d\left(t_{j}, t_{j+1}\right)$. Due to this observation the vector field $K_{0}$ can be rewritten as $K_{0}=t_{x} \tilde{X}+Y$, with

$$
\tilde{X}=\frac{\partial}{\partial t}+\frac{\partial}{\partial t_{1}}+\frac{\partial}{\partial t_{-1}}+\frac{\partial}{\partial t_{2}}+\frac{\partial}{\partial t_{-2}}+\cdots
$$

and

$$
Y=\frac{\partial}{\partial x}+d \frac{\partial}{\partial t_{1}}-d_{-1} \frac{\partial}{\partial t_{-1}}+\left(d+d_{1}\right) \frac{\partial}{\partial t_{2}}-\left(d_{-1}+d_{-2}\right) \frac{\partial}{\partial t_{-2}}+\cdots .
$$

Due to the relations $[X, \tilde{X}]=0$ and $[X, Y]=0$ we have $\tilde{X}=\left[X, K_{0}\right] \in L_{x}$; hence $Y \in L_{x}$. Therefore $L_{x}=\{X\} \oplus L_{x 1}$, where $L_{x 1}$ is the Lie algebra generated by the operators $\widetilde{X}$ and $Y$.

Lemma 1: If Eq. (12) admits a nontrivial $x$-integral, then it admits a nontrivial $x$-integral $F$ such that $\partial F / \partial x=0$.

Proof: Assume that a nontrivial $x$-integral of (12) exists. Then the Lie algebra $L_{x 1}$ is of finite dimension. One can choose a basis of $L_{x 1}$ in the form

$$
\begin{gathered}
T_{1}=\frac{\partial}{\partial x}+\sum_{k=-\infty}^{\infty} a_{1, k} \frac{\partial}{\partial t_{k}}, \\
T_{j}=\sum_{k=-\infty}^{\infty} a_{j, k} \frac{\partial}{\partial t_{k}}, \quad 2 \leq j \leq N .
\end{gathered}
$$

Thus, there exists an $x$-integral $F$ depending on $x, t, t_{1}, \ldots, t_{N-1}$, satisfying the system of equations

$$
\begin{gathered}
\frac{\partial F}{\partial x}+\sum_{k=0}^{N-1} a_{1, k} \frac{\partial F}{\partial t_{k}}=0, \\
\sum_{k=0}^{N-1} a_{j, k} \frac{\partial F}{\partial t_{k}}=0, \quad 2 \leq j \leq N .
\end{gathered}
$$

Due to the famous Jacobi theorem ${ }^{10}$ there is a change of variables $\theta_{j}=\theta_{j}\left(t, t_{1}, \ldots, t_{N-1}\right)$ that reduces the system to the form

$$
\frac{\partial F}{\partial x}+\sum_{k=0}^{N-1} \tilde{a}_{1, k} \frac{\partial F}{\partial \theta_{k}}=0
$$




$$
\frac{\partial F}{\partial \theta_{k}}=0, \quad 2 \leq j \leq N-2
$$

which is equivalent to

$$
\frac{\partial F}{\partial x}+\tilde{a}_{1, N-1} \frac{\partial F}{\partial \theta_{N-1}}=0
$$

for $F=F\left(x, \theta_{N-1}\right)$.

There are two possibilities: (1) $\tilde{a}_{1, N-1}=0$ and (2) $\tilde{a}_{1, N-1} \neq 0$. In case (1), we at once have $\partial F / \partial x=0$. In case $(2), F=x+H\left(\theta_{N-1}\right)=x+H\left(t, t_{1}, \ldots, t_{N-1}\right)$ for some function $H$. Evidently, $F_{1}$ $=D F=x+H\left(t_{1}, t_{2}, \ldots, t_{N}\right)$ is also an $x$-integral, and $F_{1}-F$ is a nontrivial $x$-integral not depending on $x$.

Below we look for $x$-integrals $F$ depending on dynamical variables $t, t_{ \pm 1}, t_{ \pm 2}, \ldots$ only (not depending on $x$ ). In other words, we study the Lie algebra generated by vector fields $\tilde{X}$ and $\tilde{Y}$, where

$$
\tilde{Y}=d \frac{\partial}{\partial t_{1}}-d_{-1} \frac{\partial}{\partial t_{-1}}+\left(d+d_{1}\right) \frac{\partial}{\partial t_{2}}-\left(d_{-1}+d_{-2}\right) \frac{\partial}{\partial t_{-2}}+\cdots .
$$

One can prove that the linear operator $Z \rightarrow D Z D^{-1}$ defines an automorphism of the characteristic Lie algebra $L_{x}$. This automorphism plays the crucial role in all of our further considerations. Further we refer to it as the shift automorphism. For instance, direct calculations show that

$$
D \tilde{X} D^{-1}=\tilde{X}, \quad D \tilde{Y} D^{-1}=-d \tilde{X}+\tilde{Y} .
$$

Lemma 2: Suppose that a vector field of the form $Z=\sum a(j)\left(\partial / \partial t_{j}\right)$ with the coefficients $a(j)$ $=a\left(j, t, t_{ \pm 1}, t_{ \pm 2}, \ldots\right)$ depending on a finite number of the dynamical variables solves an equation of the form $D Z D^{-1}=\lambda Z$. If for some $j=j_{0}$ we have $a\left(j_{0}\right) \equiv 0$, then $Z=0$.

Proof: By applying the shift automorphism to the vector field $Z$ one gets $D Z D^{-1}$ $=\Sigma D(a(j))\left(\partial / \partial t_{j+1}\right)$. Now, to complete the proof, we compare the coefficients of $\partial / \partial t_{j}$ in the equation $\Sigma D(a(j))\left(\partial / \partial t_{j+1}\right)=\lambda \Sigma a(j)\left(\partial / \partial t_{j}\right)$.

Construct an infinite sequence of multiple commutators of the vector fields $\tilde{X}$ and $\tilde{Y}$,

$$
\tilde{Y}_{1}=[\tilde{X}, \tilde{Y}], \quad \tilde{Y}_{k}=\left[\tilde{X}, \tilde{Y}_{k-1}\right] \text { for } k \geq 2
$$

Lemma 3: We have

$$
D \tilde{Y}_{k} D^{-1}=-\tilde{X}^{k}(d) \tilde{X}+\tilde{Y}_{k}, \quad k \geq 1 .
$$

Proof: We prove the statement by induction on $k$. The base of induction holds. Indeed, by (20) and (21), we have

$$
D \tilde{Y}_{1} D^{-1}=D[\tilde{X}, \tilde{Y}] D^{-1}=\left[D \tilde{X} D^{-1}, D \tilde{Y} D^{-1}\right]=[\tilde{X},-d \tilde{X}+\tilde{Y}]=-\tilde{X}(d) \tilde{X}+\tilde{Y}_{1} .
$$

Assuming Eq. (22) holds for $k=n-1$, we have

$$
D \tilde{Y}_{n} D^{-1}=\left[D \tilde{X} D^{-1}, D \tilde{Y}_{n-1} D^{-1}\right]=\left[\tilde{X},-\tilde{X}^{n-1}(d) \tilde{X}+\tilde{Y}_{n-1}\right]=-\tilde{X}^{n}(d) \tilde{X}+\tilde{Y}_{n},
$$

which finishes the proof of the lemma.

Since vector fields $X, \tilde{X}$, and $\tilde{Y}$ are linearly independent, then the dimension of Lie algebra $L_{x}$ is at least 3 . By (22), case $\tilde{Y}_{1}=0$ corresponds to $\tilde{X}(d)=0$, or $d_{t}+d_{t_{1}}=0$, which implies $d=A(t$ $\left.-t_{1}\right)$, where $A(\tau)$ is an arbitrary differentiable function of one variable. 
Assume Eq. (12) admits a nontrivial $x$-integral and $\tilde{Y}_{1} \neq 0$. Consider the sequence of the vector fields $\left\{\widetilde{Y}_{1}, \widetilde{Y}_{2}, \widetilde{Y}_{3}, \ldots\right\}$. Since $L_{x}$ is of finite dimension, then there exists a natural number $N$ such that

$$
\tilde{Y}_{N+1}=\gamma_{1} \tilde{Y}_{1}+\gamma_{2} \tilde{Y}_{2}+\cdots+\gamma_{N} \tilde{Y}_{N}, \quad N \geq 1,
$$

and $\tilde{Y}_{1}, \tilde{Y}_{2}, \ldots, \tilde{Y}_{N}$ are linearly independent. Therefore,

$$
D \widetilde{Y}_{N+1} D^{-1}=D\left(\gamma_{1}\right) D \tilde{Y}_{1} D^{-1}+D\left(\gamma_{2}\right) D \tilde{Y}_{2} D^{-1}+\cdots+D\left(\gamma_{N}\right) D \widetilde{Y}_{N} D^{-1}, \quad N \geq 1 .
$$

Due to Lemma 3 and (23) the last equation can be rewritten as

$$
\begin{aligned}
-\widetilde{X}^{N+1}(d) \tilde{X}+\gamma_{1} \tilde{Y}_{1}+\gamma_{2} \widetilde{Y}_{2}+\cdots+\gamma_{N} \widetilde{Y}_{N}= & D\left(\gamma_{1}\right)\left(-\tilde{X}(d) \tilde{X}+\widetilde{Y}_{1}\right)+D\left(\gamma_{2}\right)\left(-\widetilde{X}^{2}(d) \tilde{X}+\widetilde{Y}_{2}\right) \\
& +\cdots+D\left(\gamma_{N}\right)\left(-\tilde{X}^{N}(d) \tilde{X}+\tilde{Y}_{N}\right) .
\end{aligned}
$$

Comparing coefficients before linearly independent vector fields $\tilde{X}, \tilde{Y}_{1}, \tilde{Y}_{2}, \ldots, \widetilde{Y}_{N}$, we obtain the following system of equations:

$$
\begin{gathered}
\tilde{X}^{N+1}(d)=D\left(\gamma_{1}\right) \tilde{X}(d)+D\left(\gamma_{2}\right) \tilde{X}^{2}(d)+\cdots+D\left(\gamma_{N}\right) \tilde{X}^{N}(d), \\
\gamma_{1}=D\left(\gamma_{1}\right), \quad \gamma_{2}=D\left(\gamma_{2}\right), \ldots, \quad \gamma_{N}=D\left(\gamma_{N}\right) .
\end{gathered}
$$

Since the coefficients of the vector fields $\widetilde{Y}_{j}$ depend only on the variables $t, t_{ \pm 1}, t_{ \pm 2}, \ldots$ the factors $\gamma_{j}$ might depend only on these variables (see the proposition above). Hence the system of equations implies that all coefficients $\gamma_{k}, 1 \leq k \leq N$, are constants, and $d=d\left(t, t_{1}\right)$ is a function that satisfies the following differential equation:

$$
\tilde{X}^{N+1}(d)=\gamma_{1} \tilde{X}(d)+\gamma_{2} \tilde{X}^{2}(d)+\cdots+\gamma_{N} \tilde{X}^{N}(d),
$$

where $\tilde{X}(d)=d_{t}+d_{t_{1}}$. Using the substitution $s=t$ and $\tau=t-t_{1}$, Eq. (24) can be rewritten as

$$
\frac{\partial^{N+1} d}{\partial s^{N+1}}=\gamma_{1} \frac{\partial d}{\partial s}+\gamma_{2} \frac{\partial^{2} d}{\partial s^{2}}+\cdots+\gamma_{N} \frac{\partial^{N} d}{\partial s^{N}},
$$

which implies that

$$
d\left(t, t_{1}\right)=\sum_{k}\left(\sum_{j=0}^{m_{k}-1} \lambda_{k, j}\left(t-t_{1}\right) t^{j}\right) e^{\alpha_{k} t}
$$

for some functions $\lambda_{k, j}\left(t-t_{1}\right)$, where $\alpha_{k}$ are roots of multiplicity $m_{k}$ for a characteristic equation of (25).

Let $\alpha_{0}=0, \alpha_{1}, \ldots, \alpha_{s}$ be the distinct roots of characteristic equation (24). Equation (24) can be rewritten as

$$
\Lambda(\tilde{X}) d:=\widetilde{X}^{m_{0}}\left(\tilde{X}-\alpha_{1}\right)^{m_{1}}\left(\tilde{X}-\alpha_{2}\right)^{m_{2}} \cdots\left(\tilde{X}-\alpha_{s}\right)^{m_{s}} d=0
$$

and $m_{0}+m_{1}+\cdots+m_{s}=N+1, m_{0} \geq 1$.

Initiated by formula (19) define a map $h \rightarrow Y_{h}$ which assigns to any function $h$ $=h\left(t, t_{ \pm 1}, t_{ \pm 2}, \ldots\right)$ a vector field

$$
Y_{h}=h \frac{\partial}{\partial t_{1}}-h_{-1} \frac{\partial}{\partial t_{-1}}+\left(h+h_{1}\right) \frac{\partial}{\partial t_{2}}-\left(h_{-1}+h_{-2}\right) \frac{\partial}{\partial t_{-2}}+\cdots .
$$

For any polynomial with constant coefficients $P(\lambda)=c_{0}+c_{1} \lambda+\cdots+c_{m} \lambda^{m}$ we have the formula 


$$
P\left(a d_{\tilde{X}}\right) \tilde{Y}=Y_{P(\tilde{X}) h}, \quad \text { where } \quad a d_{X} Y=[X, Y],
$$

which establishes an isomorphism between the linear space $V$ of all solutions of Eq. (25) and the linear space $\tilde{V}=\operatorname{span}\left\{\tilde{Y}, \widetilde{Y}_{1}, \ldots, \widetilde{Y}_{N}\right\}$ of the corresponding vector fields.

Represent function (26) as the sum $d\left(t, t_{1}\right)=P\left(t, t_{1}\right)+Q\left(t, t_{1}\right)$ of the polynomial part $P\left(t, t_{1}\right)$ $=\sum_{j=0}^{m_{0}-1} \lambda_{0, j}\left(t-t_{1}\right) t^{j}$ and the "exponential" part $Q\left(t, t_{1}\right)=\sum_{k=1}^{s}\left(\sum_{j=0}^{m_{k}-1} \lambda_{k, j}\left(t-t_{1}\right) t^{j}\right) e^{\alpha_{k} t}$.

Lemma 4: Assume that Eq. (12) admits a nontrivial $x$-integral. Then one of the functions $P\left(t, t_{1}\right)$ and $Q\left(t, t_{1}\right)$ vanishes.

Proof: Assume in contrary that neither of the functions vanish. First we show that in this case algebra $L_{x}$ contains vector fields $T_{0}=Y_{A(\tau) e^{\alpha_{k} t}}$ and $T_{1}=Y_{B(\tau)}$ for some functions $A(\tau)$ and $B(\tau)$. Indeed, take $T_{0}:=\Lambda_{0}\left(a d_{\tilde{X}}\right) \tilde{Y}=Y_{\Lambda_{0}(\tilde{X}) d} \in L_{x}$, where $\Lambda_{0}(\lambda)=\Lambda(\lambda) /\left(\lambda-\alpha_{k}\right)$. Evidently the function $\widetilde{A}\left(t, t_{1}\right)=\Lambda_{0}(\widetilde{X}) d$ solves the equation $\left(\widetilde{X}-\alpha_{k}\right) \widetilde{A}\left(t, t_{1}\right)=\Lambda(\widetilde{X}) d=0$ which implies immediately that $\widetilde{A}\left(t, t_{1}\right)=A(\tau) e^{\alpha_{k} t}$. In a similar way one shows that $T_{1} \in L_{x}$. Note that due to our assumption the functions $A(\tau)$ and $B(\tau)$ cannot vanish identically.

Consider an infinite sequence of the vector fields defined as follows:

$$
T_{2}=\left[T_{0}, T_{1}\right], \quad T_{3}=\left[T_{0}, T_{2}\right], \ldots, \quad T_{n}=\left[T_{0}, T_{n-1}\right], \quad n \geq 3 .
$$

One can show that

$$
\begin{gathered}
{\left[\tilde{X}, T_{0}\right]=\alpha_{k} T_{0}, \quad\left[\tilde{X}, T_{1}\right]=0, \quad\left[\tilde{X}, T_{n}\right]=\alpha_{k}(n-1) T_{n}, \quad n \geq 2,} \\
D T_{0} D^{-1}=-A e^{\alpha_{k} t} \tilde{X}+T_{0}, \quad D T_{1} D^{-1}=-B \tilde{X}+T_{1}, \\
D T_{n} D^{-1}=T_{n}-\frac{(n-1)(n-2)}{2} \alpha_{k} A e^{\alpha_{k} t} T_{n-1}+b_{n} \tilde{X}+\sum_{k=0}^{n-2} a_{k}^{(n)} T_{k}, \quad n \geq 2 .
\end{gathered}
$$

Since algebra $L_{x}$ is of finite dimension, then there exists number $N$ such that

$$
T_{N+1}=\lambda \widetilde{X}+\mu_{0} T_{0}+\mu_{1} T_{1}+\cdots+\mu_{N} T_{N}
$$

and vector fields $\tilde{X}, T_{0}, T_{1}, \ldots, T_{N}$ are linearly independent. We have

$$
\begin{aligned}
D T_{N+1} D^{-1}= & D(\lambda) \tilde{X}+D\left(\mu_{0}\right)\left\{-A e^{\alpha_{k} t} \widetilde{X}+T_{0}\right\} \\
& +\cdots+D\left(\mu_{N}\right)\left\{T_{N}-\frac{(N-1)(N-2)}{2} \alpha_{k} A e^{\alpha_{k} t} T_{N-1}+\cdots\right\} .
\end{aligned}
$$

By comparing the coefficients before $T_{N}$ in the last equation one gets

$$
\mu_{N}-\frac{N(N-1)}{2} \alpha_{k} A(\tau) e^{\alpha_{k} t}=D\left(\mu_{N}\right)
$$

It follows that $\mu_{N}$ is a function of variable $t$ only. Also, by applying $a d_{\tilde{X}}$ to both sides of Eq. (29), one gets

$$
N \alpha_{k} T_{N+1}=\left[\tilde{X}, T_{N+1}\right]=\tilde{X}(\lambda) \tilde{X}+\left(\tilde{X}\left(\mu_{0}\right)+\mu_{0} \alpha_{k}\right) T_{0}+\cdots+\left(\tilde{X}\left(\mu_{N}\right)+\mu_{N}(N-1) \alpha_{k}\right) T_{N} .
$$

Again, by comparing coefficients before $T_{N}$, we have

$$
N \alpha_{k} \mu_{N}=\tilde{X}\left(\mu_{N}\right)+(N-1) \alpha_{k} \mu_{N}, \text { i. e. }, \quad \tilde{X}\left(\mu_{N}\right)=\alpha_{k} \mu_{N} .
$$

Therefore, $\mu_{N}=A_{1} e^{\alpha_{k} t}$, where $A_{1}$ is some nonzero constant, and thus $A(\tau) e^{\alpha_{k} t}=A_{2} e^{\alpha_{k} t}-A_{2} e^{\alpha_{k} t_{1}}$. Here $A_{2}$ is some constant. We have $T_{0}=A_{2} e^{\alpha_{k} t} \widetilde{X}-A_{2} S_{0}$, where 


$$
S_{0}=\sum_{j=-\infty}^{\infty} e^{\alpha_{k} t_{j}} \frac{\partial}{\partial t_{j}} .
$$

Also,

$$
\left[\tilde{X}, S_{0}\right]=\alpha_{k} S_{0}, \quad D S_{0} D^{-1}=S_{0} .
$$

Consider a new sequence of vector fields,

$$
P_{1}=S_{0}, \quad P_{2}=\left[T_{1}, S_{0}\right], \quad P_{3}=\left[T_{1}, P_{2}\right], \quad P_{n}=\left[T_{1}, P_{n-1}\right], \quad n \geq 3 .
$$

One can show that

$$
\left[\tilde{X}, P_{n}\right]=\alpha_{k} P_{n}, \quad D P_{n} D^{-1}=P_{n}-\alpha_{k}(n-1) B P_{n-1}+b_{n} \tilde{X}+a_{n} S_{0}+\sum_{j=2}^{n-2} a_{j}^{(n)} P_{j}, \quad n \geq 2 .
$$

Since algebra $L_{x}$ is of finite dimension, then there exists number $M$ such that

$$
P_{M+1}=\lambda^{*} \tilde{X}+\mu_{2}^{*} P_{2}+\cdots+\mu_{M}^{*} P_{M},
$$

and fields $\tilde{X}, P_{2}, \ldots, P_{M}$ are linearly independent. Thus,

$$
D P_{M+1} D^{-1}=D\left(\lambda^{*}\right) \tilde{X}+D\left(\mu_{2}^{*}\right)\left\{P_{2}+\cdots\right\}+\cdots+D\left(\mu_{M}^{*}\right)\left\{P_{M}-\alpha_{k}(M-1) B P_{M-1}+\cdots\right\} .
$$

We compare the coefficients before $P_{M}$ in the last equation and get

$$
\mu_{M}^{*}-M \alpha_{k} B(\tau)=D\left(\mu_{M}^{*}\right),
$$

which implies that $\mu_{M}^{*}$ is a function of variable $t$ only. Also, by applying $a d_{\tilde{X}}$ to both sides of (30), one gets

$$
\alpha_{k} P_{M+1}=\left[\tilde{X}, P_{M+1}\right]=\tilde{X}\left(\lambda^{*}\right) \tilde{X}+\left(\tilde{X}\left(\mu_{2}^{*}\right)+\alpha_{k} \mu_{2}^{*}\right) P_{2}+\cdots+\left(\tilde{X}\left(\mu_{M}^{*}\right)+\alpha_{k} \mu_{M}^{*}\right) P_{M} .
$$

Again, we compare the coefficients before $P_{M}$ and have $\alpha_{k} \mu_{M}^{*}(t)=\tilde{X}\left(\mu_{M}^{*}(t)\right)+\alpha_{k} \mu_{M}^{*}(t)$, which implies that $\mu_{M}^{*}$ is a constant. It follows then from (31) that $B(\tau)=0$. This contradiction shows that our assumption that both functions are not identically zero was wrong.

\section{MULTIPLE ZERO ROOT}

In this section we assume that Eq. (12) admits a nontrivial $x$-integral and that $\alpha_{0}=0$ is a root of the characteristic polynomial $\Lambda(\lambda)$. Then, due to Lemma 4 , zero is the only root and therefore $\Lambda(\lambda)=\lambda^{m+1}$. It follows from formula (26) with $m_{0}=m+1$ that

$$
d\left(t, t_{1}\right)=a(\tau) t^{m}+b(\tau) t^{m-1}+\cdots, \quad m=m_{0}-1 \geq 0 .
$$

The case $m=0$ corresponds to a very simple equation, $t_{1 x}=t_{x}+A\left(t-t_{1}\right)$, which is easily solved in quadratures, so we concentrate on the case $m \geq 1$. For this case the characteristic algebra $L_{x}$ contains a vector field $T=Y_{\widetilde{\kappa}}$ with

$$
\widetilde{\kappa}=a(\tau) t+\frac{1}{m} b(\tau)
$$

Indeed,

$$
T=\frac{1}{m !} a d_{\tilde{X}}^{m-1} \tilde{Y}=Y_{\tilde{\kappa}}
$$

Introduce a sequence of multiple commutators defined as follows: 


$$
T_{0}=\tilde{X}, \quad T_{1}=\left[T, T_{0}\right]=Y_{-a(\tau)}, \quad T_{k+1}=\left[T, T_{k}\right], \quad k \geq 0, \quad T_{k, 0}=\left[T_{0}, T_{k}\right] .
$$

Note that $T_{1,0}=0$. We will see below that the linear space spanned by this sequence is not invariant under the action of the shift automorphism $Z \rightarrow D Z D^{-1}$ introduced above. We extend the sequence to provide the invariance property. We define $T_{\alpha}$ with the multi-index $\alpha$. For any sequence $\alpha$ $=k, 0, i_{1}, i_{2}, \ldots, i_{n-1}, i_{n}$, where $k$ is any natural number, $i_{j} \in\{0 ; 1\}$, denote

$$
\begin{gathered}
T_{\alpha}= \begin{cases}{\left[T_{0}, T_{k, 0, i_{1}, \ldots, i_{n-1}}\right]} & \text { if } i_{n}=0 \\
{\left[T, T_{k, 0, i_{1}, \ldots, i_{n-1}}\right]} & \text { if } i_{n}=1,\end{cases} \\
m(\alpha)= \begin{cases}k & \text { if } \alpha=k \\
k & \text { if } \alpha=k, 0 \\
k+i_{1}+\ldots+i_{n} & \text { if } \alpha=k, 0, i_{1}, \ldots, i_{n},\end{cases} \\
l(\alpha)=k+n+1-m(\alpha) .
\end{gathered}
$$

The multi-index $\alpha$ is characterized by two quantities, $m(\alpha)$ and $l(\alpha)$, which allow to order partially the sequence $\left\{T_{\alpha}\right\}$. We have

$$
D T_{0} D^{-1}=T_{0}, \quad D T D^{-1}=T-\widetilde{\kappa} T_{0}, \quad D T_{1} D^{-1}=T_{1}+a T_{0} .
$$

One can prove by induction on $k$ that

$$
D T_{k} D^{-1}=T_{k}+a T_{k-1}-\widetilde{\kappa} \sum_{m(\beta)=k-1} T_{\beta}+\sum_{m(\beta) \leq k-2} \eta(k, \beta) T_{\beta} .
$$

In general, for any $\alpha$,

$$
D T_{\alpha} D^{-1}=T_{\alpha}+\sum_{m(\beta) \leq m(\alpha)-1} \eta(\alpha, \beta) T_{\beta} .
$$

We can choose a system $P$ of linearly independent vector fields in the following way:

(1) $T$ and $T_{0}$ are linearly independent. We take them into $P$.

(2) We check whether $T, T_{0}$, and $T_{1}$ are linearly independent or not. If they are dependent, then $P=\left\{T, T_{0}\right\}$ and $T_{1}=\mu T+\lambda T_{0}$ for some functions $\mu$ and $\lambda$.

(3) If $T, T_{0}$, and $T_{1}$ are linearly independent, then we check whether $T, T_{0}$, and $T_{1}, T_{2}$ are linearly independent or not. If they are dependent, then $P=\left\{T, T_{0}, T_{1}\right\}$.

(4) If $T, T_{0}, T_{1}$, and $T_{2}$ are linearly independent, we add vector fields $T_{\beta}, m(\beta)=2, \beta \in I_{2}$ (actually, by definition $I_{2}$ is the collection of such $\beta$ ), in such a way that $J_{2}$ $:=\left\{T, T_{0}, T_{1}, T_{2}, \cup_{\beta \in I_{2}} T_{\beta}\right\}$ is a system of linearly independent vector fields and for any $T_{\gamma}$ with $m(\gamma) \leq 2$ we have $T_{\gamma}=\Sigma_{T_{\beta} \in J_{2}} \mu(\gamma, \beta) T_{\beta}$.

(5) We check whether $T_{3} \cup J_{2}$ is a linearly independent system. If it is not, then $P$ consists of all elements from $J_{2}$, and $T_{3}=\Sigma_{T_{\beta} \in J_{2}} \mu(\gamma, \beta) T_{\beta}$. If it is, then to the system $T_{3} \cup J_{2}$ we add vector fields $T_{\beta}, m(\beta)=3, \beta \in I_{3}$, in such a way that $J_{3}:=\left\{T_{3}, J_{2}, \cup_{\beta \in I_{3}} T_{\beta}\right\}$ is a system of linearly independent vector fields and for any $T_{\gamma}$ with $m(\gamma) \leq 3$ we have $T_{\gamma}=\Sigma_{T_{\beta} \in J_{3}} \mu(\gamma, \beta) T_{\beta}$.

We continue the construction of system $P$. Since $L_{x}$ is of finite dimension, then there exists such a natural number $N$ such that we have the following:

(i) $T_{k} \in P, k \leq N$.

(ii) $\quad m(\beta) \leq N$ for any $T_{\beta} \in P$.

(iii) For any $T_{\gamma}$ with $m(\gamma) \leq N$ we have $T_{\gamma}=\Sigma_{T_{\beta} \in P, m(\beta) \leq m(\gamma)} \mu(\gamma, \beta) T_{\beta}$ and also $T_{N+1}=\mu(N+1, N) T_{N}+\Sigma_{T_{\beta} \in P, m(\beta) \leq N} \mu(N+1, \beta) T_{\beta}$. 
We then have the following:

(iv) For any vector field $T_{\alpha}$ with $m(\alpha)=N$ that does not belong to $P$, the coefficient $\mu(\alpha, N)$ before $T_{N}$ in the expansion

$$
T_{\alpha}=\mu(\alpha, N) T_{N}+\sum_{T_{\beta} \in P} \mu(\alpha, \beta) T_{\beta}
$$

is constant. Indeed, by (34),

$$
D T_{\alpha} D^{-1}=T_{\alpha}+\sum_{m(\beta) \leq N-1} \eta(\alpha, \beta) T_{\beta}=\mu(\alpha, N) T_{N}+\sum_{T_{\beta} \in P} \mu(\alpha, \beta) T_{\beta}+\sum_{m(\beta) \leq N-1} \eta(\alpha, \beta) T_{\beta} .
$$

From (35) we also have

$$
\begin{aligned}
D T_{\alpha} D^{-1}= & D(\mu(\alpha, N)) D T_{N} D^{-1}+\sum_{T_{\beta} \in P} D(\mu(\alpha, \beta)) D T_{\beta} D^{-1}=D(\mu(\alpha, N))\left\{T_{N}+\cdots\right\} \\
& +\sum_{T_{\beta} \in P} D(\mu(\alpha, \beta))\left\{T_{\beta}+\cdots\right\} .
\end{aligned}
$$

By comparing the coefficients before $T_{N}$ in these two expressions for $D T_{\alpha} D^{-1}$, we have

$$
\mu(\alpha, N)=D(\mu(\alpha, N)),
$$

which implies that $\mu(\alpha, N)$ is a constant indeed.

Lemma 5: We have $a(\tau)=c_{0} \tau+c_{1}$, where $c_{0}$ and $c_{1}$ are some constants.

Proof: Since

$$
T_{N+1}=\mu(N+1, N) T_{N}+\sum_{T_{\beta} \in P} \mu(N+1, \beta) T_{\beta},
$$

then

$$
D T_{N+1} D^{-1}=D(\mu(N+1, N))\left\{T_{N}+\cdots\right\}+\sum_{T_{\beta} \in P} D(\mu(N+1, \beta))\left\{T_{\beta}+\cdots\right\} .
$$

On the other hand,

$$
D T_{N+1} D^{-1}=T_{N+1}+a T_{N}-\widetilde{\kappa} \sum_{m(\beta)=N} T_{\beta}+\sum_{m(\beta) \leq N-1} \eta(N+1, \beta) T_{\beta} .
$$

We compare the coefficients before $T_{N}$ in the last two expressions. For $N \geq 0$ the equation is

$$
\mu(N+1, N)+a-\widetilde{\kappa} \sum_{T_{\beta} \in P, m(\beta)=N} \mu(\beta, N)=D(\mu(N+1, N)) .
$$

Denote by $c=-\Sigma_{T_{\beta} \in P, m(\beta)=N} \mu(\beta, N)$ and by $\mu_{N}=\mu(N+1, N)$. By property (iv), $c$ is a constant. It follows from (36) that $\mu_{N}$ is a function of variables $t$ and $n$ only. Therefore,

$$
a(\tau)+c\left(a(\tau) t+\frac{1}{m} b(\tau)\right)=\mu_{N}\left(t_{1}, n+1\right)-\mu_{N}(t, n) .
$$

By differentiating both sides of the equation with respect to $t$ and then $t_{1}$, we have

$$
-a^{\prime \prime}(\tau)-c\left(a^{\prime \prime}(\tau) t+a^{\prime}(\tau)+\frac{1}{m} b^{\prime \prime}(\tau)\right)=0,
$$

which implies that $a^{\prime \prime}(\tau)=0$, or the same, $a(\tau)=c_{0} \tau+c_{1}$ for some constants $c_{0}$ and $c_{1}$.

Vector fields $T_{1}$ and $T$ in new variables are rewritten as 


$$
\begin{gathered}
T_{1}=\sum_{j=-\infty}^{\infty} a\left(\tau_{j}\right) \frac{\partial}{\partial \tau_{j}}, \\
T=-\sum_{j=-\infty}^{\infty}\left\{a\left(\tau_{j}\right) t_{j}+\frac{1}{m} b\left(\tau_{j}\right)\right\} \frac{\partial}{\partial \tau_{j}}=-\sum_{j=-\infty}^{\infty}\left\{a\left(\tau_{j}\right)\left(t+\rho_{j}\right)+\frac{1}{m} b\left(\tau_{j}\right)\right\} \frac{\partial}{\partial \tau_{j}} \\
=-t T_{1}-\sum_{j=-\infty}^{\infty}\left\{a\left(\tau_{j}\right) \rho_{j}+\frac{1}{m} b\left(\tau_{j}\right)\right\} \frac{\partial}{\partial \tau_{j}},
\end{gathered}
$$

where

$$
\rho_{j}= \begin{cases}-\tau-\tau_{1}-\cdots-\tau_{j-1} & \text { if } j \geq 1 \\ 0 & \text { if } j=0 \\ \tau_{-1}+\tau_{-2}+\cdots+\tau_{j} & \text { if } j \leq-1\end{cases}
$$

The following two lemmas are to be useful.

Lemma 6: If the Lie algebra generated by the vector fields $S_{0}=\sum_{j=-\infty}^{\infty} \partial / \partial w_{j}$ and $P$ $=\sum_{j=-\infty}^{\infty} c\left(w_{j}\right)\left(\partial / \partial w_{j}\right)$ is of finite dimension, then $c(w)$ is one of the following forms:

(1) $c(w)=c_{2}+c_{3} e^{\lambda w}+c_{4} e^{-\lambda w}, \lambda \neq 0$, and

(2) $c(w)=c_{2}+c_{3} w+c_{4} w^{2}$, where $c_{2}-c_{4}$ are some constants.

Proof: Introduce vector fields

$$
S_{1}=\left[S_{0}, P\right], \quad S_{2}=\left[S_{0}, S_{1}\right], \ldots, \quad S_{n}=\left[S_{0}, S_{n-1}\right], \quad n \geq 3 .
$$

Clearly, we have

$$
S_{n}=\sum_{j=-\infty}^{\infty} c^{(n)}\left(w_{j}\right) \frac{\partial}{\partial w_{j}}, \quad n \geq 1 .
$$

Since all vector fields $S_{n}$ are elements of $L_{x}$ and $L_{x}$ is of finite dimension, then there exists a natural number $N$ such that

$$
S_{N+1}=\mu_{N} S_{N}+\mu_{N-1} S_{N-1}+\cdots+\mu_{1} S_{1}+\mu_{0} P+\mu S_{0},
$$

and $S_{0}, P, S_{1}, \ldots, S_{N}$ are linearly independent. (Note that we may assume that $S_{0}$ and $P$ are linearly independent). Since $D S_{0} D^{-1}=S_{0}, D P D^{-1}=P$, and $D S_{n} D^{-1}=S_{n}$ for any $n \geq 1$, then it follows from (40) that

$$
S_{N+1}=D\left(\mu_{N}\right) S_{N}+D\left(\mu_{N-1}\right) S_{N-1}+\cdots+D\left(\mu_{1}\right) S_{1}+D\left(\mu_{0}\right) P+D(\mu) S_{0}
$$

and together with (40), it implies that $\mu, \mu_{0}, \mu_{1}, \ldots, \mu_{N}$ are all constants.

By comparing the coefficients before $\partial / \partial w$ in (40) one gets, with the help of (39), the following equality:

$$
c^{(N+1)}(w)=\mu_{N} c^{(N)}(w)+\cdots+\mu_{1} c^{\prime}(w)+\mu_{0} c(w)+\mu .
$$

Thus, $c(w)$ is a solution of the nonhomogeneous linear differential equation with constant coefficient whose characteristic polynomial is

$$
\Lambda(\lambda)=\lambda^{N+1}-\mu_{N} \lambda^{N}-\cdots-\mu_{1} \lambda-\mu_{0} .
$$

Denote by $\beta_{1}, \beta_{2}, \ldots, \beta_{t}$ the characteristic roots and by $m_{1}, m_{2}, \ldots, m_{t}$ their multiplicities. Following are the possibilities: 
(i) There exists a nonzero characteristic root, say, $\beta_{1}$, and its multiplicity $m_{1} \geq 2$.

(ii) There exists zero characteristic root, say, $\beta_{1}$, and $m_{1} \geq 3, \mu=0$ or $m_{1} \geq 2, \mu \neq 0$.

(iii) There are two distinct characteristic roots, say, $\beta_{1}$ and $\beta_{2}$ with $\beta_{1} \neq 0$ and $\beta_{2}=0$.

(iv) There are two nonzero distinct characteristic roots, say, $\beta_{1}$ and $\beta_{2}$.

In case (i), consider

$$
\Lambda_{1}(\lambda)=\frac{\Lambda(\lambda)}{\lambda-\beta_{1}} \quad \text { and } \quad \Lambda_{1}^{(2)}(\lambda)=\frac{\Lambda(\lambda)}{\left(\lambda-\beta_{1}\right)^{2}} .
$$

Then $\Lambda_{1}\left(S_{0}\right) c(w)=\alpha_{1} e^{\beta_{1} w}+\alpha_{2}$ and $\Lambda_{1}^{(2)}\left(S_{0}\right) c(w)=\left(\alpha_{3} w+\alpha_{4}\right) e^{\beta_{1} w}+\alpha_{5}$, where $\alpha_{j}, 1 \leq j \leq 5$, are some constants with $\alpha_{1} \neq 0$ and $\alpha_{3} \neq 0$. We have

$$
\begin{aligned}
\Lambda_{1}\left(a d_{S_{0}}\right) P & =\sum_{j=-\infty}^{\infty}\left(\alpha_{1} e^{\beta_{1} w_{j}}+\alpha_{2}\right) \frac{\partial}{\partial w_{j}}=\alpha_{1}\left(\sum_{j=-\infty}^{\infty} e^{\beta_{1} w_{j}} \frac{\partial}{\partial w_{j}}\right)+\alpha_{2} S_{0}=\alpha_{1} P_{1}+\alpha_{2} S_{0}, \\
\Lambda_{1}^{(2)}\left(a d_{S_{0}}\right) P & =\sum_{j=-\infty}^{\infty}\left(\left(\alpha_{3} w_{j}+\alpha_{4}\right) e^{\beta_{1} w_{j}}+\alpha_{5}\right) \frac{\partial}{\partial w_{j}}=\alpha_{3}\left(\sum_{j=-\infty}^{\infty} w_{j} e^{\beta_{1} w_{j}} \frac{\partial}{\partial w_{j}}\right)+\alpha_{4} P_{1}+\alpha_{5} S_{0} \\
& =\alpha_{3} P_{2}+\alpha_{4} P_{1}+\alpha_{5} S_{0}
\end{aligned}
$$

are elements from $L_{x}$ and therefore vector fields $P_{1}=\sum_{j=-\infty}^{\infty} e^{\beta_{1} w_{j}}\left(\partial / \partial w_{j}\right)$ and $P_{2}$ $=\sum_{j=-\infty}^{\infty} w_{j} e^{\beta_{1} w_{j}}\left(\partial / \partial w_{j}\right)$ belong to $L_{x}$. Since $P_{1}$ and $P_{2}$ generate an infinite dimensional Lie algebra $L_{x}$, then case (i) fails to be true.

In case (ii), consider

$$
\Lambda_{1}^{(3)}(\lambda)=\frac{\Lambda(\lambda)}{\lambda^{3}} \quad \text { and } \quad \Lambda_{1}^{(2)}(\lambda)=\frac{\Lambda(\lambda)}{\lambda^{2}} \quad \text { if } \mu=0
$$

or

$$
\Lambda_{1}^{(3)}(\lambda)=\frac{\Lambda(\lambda)}{\lambda^{2}} \quad \text { and } \quad \Lambda_{1}^{(2)}(\lambda)=\frac{\Lambda(\lambda)}{\lambda} \quad \text { if } \mu \neq 0
$$

We have

$$
\Lambda_{1}^{(3)}\left(S_{0}\right) c(w)=\alpha_{1} w^{3}+\alpha_{2} w^{2}+\alpha_{3} w+\alpha_{4} \quad \text { and } \quad \Lambda_{1}^{(2)}\left(S_{0}\right) c(w)=\alpha_{5} w^{2}+\alpha_{6} w+\alpha_{7},
$$

where $\alpha_{j}, 1 \leq j \leq 7$, are some constants with $\alpha_{1} \neq 0$ and $\alpha_{5} \neq 0$. Straightforward calculations show that vector fields

$$
\begin{aligned}
\Lambda_{1}^{(3)}\left(a d_{S_{0}}\right) P & =\sum_{j=-\infty}^{\infty}\left(\alpha_{1} w_{j}^{3}+\alpha_{2} w_{j}^{2}+\alpha_{3} w_{j}+\alpha_{4}\right) \frac{\partial}{\partial w_{j}} \quad \text { and } \Lambda_{1}^{(2)}\left(a d_{S_{0}}\right) P \\
& =\sum_{j=-\infty}^{\infty}\left(\alpha_{5} w_{j}^{2}+\alpha_{6} w_{j}+\alpha_{7}\right) \frac{\partial}{\partial w_{j}}
\end{aligned}
$$

generate an infinite dimensional Lie algebra. It proves that case (ii) fails to be true.

In case (iii), consider

$$
\Lambda_{1}(\lambda)=\frac{\Lambda(\lambda)}{\lambda-\beta_{1}} \quad \text { and } \quad \Lambda_{2}(\lambda)=\frac{\Lambda(\lambda)}{\lambda}
$$

We have 


$$
\Lambda_{1} c(w)=\alpha_{1} e^{\beta_{1} w}+\alpha_{2} \quad \text { and } \quad \Lambda_{2} c(w)=\alpha_{3} w+\alpha_{4} \quad \text { if } \mu=0
$$

or

$$
\Lambda_{1}\left(S_{0}\right) c(w)=\alpha_{1} e^{\beta_{1} w}+\alpha_{2} \quad \text { and } \quad \Lambda_{2}\left(S_{0}\right) c(w)=\alpha_{5} w^{2}+\alpha_{6} w+\alpha_{7} \quad \text { if } \mu \neq 0,
$$

where $\alpha_{j}, 1 \leq j \leq 7$, are constants with $\alpha_{1} \neq 0, \alpha_{3} \neq 0$, and $\alpha_{5} \neq 0$. Since vector fields $\Lambda_{1}\left(a d_{S_{0}}\right) P$ and $\Lambda_{2}\left(a d_{S_{0}}\right) P$ generate an infinite dimensional Lie algebra, then case (iii) also fails to exist.

In case (iv), consider

$$
\Lambda_{1}(\lambda)=\frac{\Lambda(\lambda)}{\lambda-\beta_{1}} \quad \text { and } \quad \Lambda_{2}(\lambda)=\frac{\Lambda(\lambda)}{\lambda-\beta_{2}} .
$$

We have $\Lambda_{1}\left(S_{0}\right) c(w)=\alpha_{1} e^{\beta_{1} w}+\alpha_{2}$ and $\Lambda_{2}\left(S_{0}\right) c(w)=\alpha_{3} e^{\beta_{2} w}+\alpha_{4}$, where $\alpha_{1} \neq 0, \alpha_{2}, \alpha_{3} \neq 0$, and $\alpha_{4}$ are some constants. Note that

$$
\Lambda_{1}\left(a d_{S_{0}}\right) P=\alpha_{1}\left(\sum_{j=-\infty}^{\infty} e^{\beta_{1} w_{j}} \frac{\partial}{\partial w_{j}}\right)+\alpha_{2} S_{0} \quad \text { and } \quad \Lambda_{2}\left(a d_{S_{0}}\right) P=\alpha_{3}\left(\sum_{j=-\infty}^{\infty} e^{\beta_{2} w_{j}} \frac{\partial}{\partial w_{j}}\right)+\alpha_{4} S_{0}
$$

and vector fields $\sum_{j=-\infty}^{\infty} e^{\beta_{1} w_{j}}\left(\partial / \partial w_{j}\right)$ and $\sum_{j=-\infty}^{\infty} e^{\beta_{2} w_{j}}\left(\partial / \partial w_{j}\right)$ generate an infinite dimensional Lie algebra if $\beta_{1}+\beta_{2} \neq 0$.

It follows from (i)-(iv) that $c(w)$ is one of the following forms:

(1) $c(w)=c_{2}+c_{3} e^{\lambda w}+c_{4} e^{-\lambda w}, \lambda \neq 0$.

(2) $c(w)=c_{2}+c_{3} w+c_{4} w^{2}$, where $c_{2}-c_{4}$ are some constants.

Lemma 7: If the Lie algebra generated by the vector fields $S_{0}=\sum_{j=-\infty}^{\infty} \partial / \partial w_{j}$, $Q=\sum_{j=-\infty}^{\infty} q\left(w_{j}\right)\left(\partial / \partial w_{j}\right)$, and $S_{1}=\sum_{j=-\infty}^{\infty}\left\{\tilde{\rho}_{j}+\tilde{b}\left(w_{j}\right)\right\}\left(\partial / \partial w_{j}\right)$ is of finite dimension, then $q(w)$ is a constant function.

Proof: It follows from Lemma 6 that

(1) $q(w)=c_{2}+c_{3} w+c_{4} w^{2}$ or

(2) $q(w)=c_{2}+c_{3} e^{\lambda w}+c_{4} e^{-\lambda w}, \lambda \neq 0$,

where $c_{2}-c_{4}$ are some constants.

Consider case (1). We have

$$
\left[S_{0}, Q\right]=c_{3} \sum_{j=-\infty}^{\infty} \frac{\partial}{\partial w_{j}}+2 c_{4} \sum_{j=-\infty}^{\infty} w_{j} \frac{\partial}{\partial w_{j}}=c_{3} S_{0}+2 c_{4} \sum_{j=-\infty}^{\infty} w_{j} \frac{\partial}{\partial w_{j}} .
$$

If $c_{4} \neq 0$, then $\sum_{j=-\infty}^{\infty} w_{j}\left(\partial / \partial w_{j}\right) \in L_{x}$ and $\sum_{j=-\infty}^{\infty} w_{j}^{2}\left(\partial / \partial w_{j}\right) \in L_{x}$.

If $c_{4}=0$ and $c_{3} \neq 0$, then $\sum_{j=-\infty}^{\infty} w_{j}\left(\partial / \partial w_{j}\right)=\left(1 / c_{3}\right)\left(Q-c_{2} S_{0}\right) \in L_{x}$.

If $c_{3}=c_{4}=0$, then $q(w)=c_{2}$ and there is nothing to prove.

Assume $c_{4}^{2}+c_{3}^{2} \neq 0$. Denote by $P=\sum_{j=-\infty}^{\infty} w_{j}\left(\partial / \partial w_{j}\right)$. Construct the vector fields

$$
P_{1}=\left[P, S_{1}\right], \quad P_{n}=\left[P, P_{n-1}\right], \quad n \geq 2 .
$$

We have

$$
\begin{gathered}
D S_{0} D^{-1}=S_{0}, \\
D S_{1} D^{-1}=S_{1}-\left(e^{w}-\widetilde{c}\right) S_{0}, \\
D P D^{-1}=P,
\end{gathered}
$$




$$
\begin{gathered}
D P_{1} D^{-1}=P_{1}+\left(-w e^{w}+e^{w}-\widetilde{c}\right) S_{0}, \\
D P_{2} D^{-1}=P_{2}+\left(-w^{2} e^{w}+w e^{w}-e^{w}+\widetilde{c}\right) S_{0} .
\end{gathered}
$$

In general,

$$
D P_{n} D^{-1}=P_{n}+\left(-w^{n} e^{w}+R_{n-1}(w) e^{w}+c_{n}\right) S_{0}, \quad n \geq 3,
$$

where $R_{n-1}$ is a polynomial of degree $n-1$ and $c_{n}$ is a constant. Since $L_{x}$ is of finite dimension, then there exists a natural number $N$ such that

$$
P_{N+1}=\mu_{N} P_{N}+\cdots+\mu_{1} P_{1}+\mu_{0} S_{0},
$$

and $S_{0}, P_{1}, \ldots, P_{N}$ are linearly independent. Thus

$$
D P_{N+1} D^{-1}=D\left(\mu_{N}\right) D P_{N} D^{-1}+\cdots+D\left(\mu_{1}\right) D P_{1} D^{-1}+D\left(\mu_{0}\right) S_{0},
$$

or the same,

$$
\begin{aligned}
& \mu_{N} P_{N}+\cdots+\mu_{1} P_{1}+\mu_{0} S_{0}+\left(-w^{N+1} e^{w}+R_{N}(w) e^{w}+c_{N+1}\right) S_{0} \\
& =D\left(\mu_{N}\right)\left\{P_{N}+\left(-w^{N} e^{w}+R_{N-1}(w) e^{w}+c_{N}\right) S_{0}\right\}+\cdots+D\left(\mu_{1}\right)\left\{P_{1}+\left(-w e^{w}+e^{w}-\widetilde{c}\right) S_{0}\right\} \\
& \quad+D\left(\mu_{0}\right) S_{0} .
\end{aligned}
$$

By comparing the coefficients before $P_{N}, \ldots, P_{1}$ we have

$$
\mu_{N}=D\left(\mu_{N}\right), \ldots, \mu_{1}=D\left(\mu_{1}\right)
$$

which implies that $\mu_{N}, \ldots, \mu_{1}$ are all constants. By comparing the coefficients before $S_{0}$ we have

$$
\begin{aligned}
\mu_{0}-w^{N+1} e^{w}+R_{N}(w) e^{w}+c_{N+1}= & \mu_{N}\left(-w^{N} e^{w}+R_{N-1}(w) e^{w}+c_{N}\right)+\cdots+\mu_{1}\left(-w e^{w}+e^{w}-\tilde{c}\right) \\
& +D\left(\mu_{0}\right) .
\end{aligned}
$$

The last equality shows that $D\left(\mu_{0}\right)-\mu_{0}$ is a function of $w$ only. Thus $D\left(\mu_{0}\right)-\mu_{0}$ is a constant; we denote it by $d_{0}$. The last equality becomes a contradictory one:

$$
w^{N+1} e^{w}=R_{N}(w) e^{w}+c_{N+1}-\mu_{N}\left(-w^{N} e^{w}+R_{N-1}(w) e^{w}+c_{N}\right)-\cdots-\mu_{1}\left(-w e^{w}+e^{w}-\tilde{c}\right)-d_{0} .
$$

This contradiction proves that $c_{3}^{2}+c_{4}^{2}=0$, i.e., $c_{3}=c_{4}=0$ in case (1). Therefore, $q(w)=c_{2}$.

Consider case (2). Since

$$
\begin{aligned}
& {\left[S_{0}, Q\right]=\lambda c_{3} \sum_{j=-\infty}^{\infty} e^{\lambda w_{j}} \frac{\partial}{\partial w_{j}}-\lambda c_{4} \sum_{j=-\infty}^{\infty} e^{-\lambda w_{j}} \frac{\partial}{\partial w_{j}},} \\
& {\left[S_{0},\left[S_{0}, Q\right]\right]=\lambda^{2} c_{3} \sum_{j=-\infty}^{\infty} e^{\lambda w_{j}} \frac{\partial}{\partial w_{j}}+\lambda^{2} c_{4} \sum_{j=-\infty}^{\infty} e^{-\lambda w_{j}} \frac{\partial}{\partial w_{j}},}
\end{aligned}
$$

then vector fields $Q_{\lambda}=c_{3} \sum_{j=-\infty}^{\infty} e^{\lambda w_{j}}\left(\partial / \partial w_{j}\right)$ and $Q_{-\lambda}=c_{4} \sum_{j=-\infty}^{\infty} e^{-\lambda w_{j}}\left(\partial / \partial w_{j}\right)$ both belong to $L_{x}$. We have $D Q_{\lambda} D^{-1}=Q_{\lambda}$ and $D Q_{-\lambda} D^{-1}=Q_{-\lambda}$.

Assume $c_{3} \neq 0$. Construct vector fields

$$
Q_{1}=\left[Q_{\lambda}, S_{1}\right], \quad Q_{n}=\left[Q_{\lambda}, Q_{n-1}\right], \quad n \geq 2 .
$$

Direct calculations show that

$$
D Q_{1} D^{-1}=Q_{1}-c_{3} e^{(1+\lambda) w} S_{0}+\left(e^{w}-\widetilde{c}\right) \lambda Q_{\lambda},
$$




$$
D Q_{2} D^{-1}=Q_{2}-c_{3}^{2}(1+\lambda) e^{(1+2 \lambda) w} S_{0}+2 \lambda c_{3} e^{(1+\lambda) w} Q_{\lambda} .
$$

It can be proved by induction on $n$ that

$$
D Q_{n} Q^{-1}=Q_{n}-p_{n} S_{0}+q_{n} Q_{\lambda}, \quad n \geq 2,
$$

where

$$
\begin{aligned}
& p_{n}=c_{3}^{n}(1+\lambda)(1+2 \lambda) \cdots(1+(n-1) \lambda) e^{(1+n \lambda) w}, \\
& q_{n}=n c_{3}^{n-1} \lambda(1+\lambda) \cdots(1+(n-2) \lambda) e^{(1+(n-1) \lambda) w} .
\end{aligned}
$$

Since $L_{x}$ is of finite dimension, there exists such a natural number $N$ that

$$
Q_{N+1}=\mu_{N} Q_{N}+\cdots+\mu_{1} Q_{1}+\mu_{\lambda} Q_{\lambda}+\mu_{0} S_{0},
$$

and $S_{0}, Q_{\lambda}, Q_{1}, \ldots, Q_{N}$ are linearly independent. Then

$$
D Q_{N+1} D^{-1}=D\left(\mu_{N}\right) D Q_{N} D^{-1}+\cdots+D\left(\mu_{0}\right) D S_{0} D^{-1}
$$

or

$$
\begin{gathered}
\mu_{N} Q_{N}+\cdots+\mu_{1} Q_{1}+\mu_{\lambda} Q_{\lambda}+\mu_{0} S_{0}-p_{N+1} S_{0}+q_{N+1} Q_{\lambda}=D\left(\mu_{N}\right)\left\{Q_{N}-p_{N} S_{0}+q_{N} Q_{\lambda}\right\} \\
+\cdots+D\left(\mu_{1}\right)\left\{Q_{1}-p_{1} S_{0}+q_{1} Q_{\lambda}\right\}+D\left(\mu_{\lambda}\right) Q_{\lambda}+D\left(\mu_{0}\right) S_{0} .
\end{gathered}
$$

By comparing the coefficients before $Q_{N}, \ldots, Q_{1}$, we have that $\mu_{k}, 1 \leq k \leq N$, are all constants. Comparing coefficients before $S_{0}$ gives

$$
\mu_{0}-p_{N+1}=-\mu_{N} p_{N}-\cdots-\mu_{2} p_{2}-\mu_{1} p_{1}+D\left(\mu_{0}\right) .
$$

Since $p_{k}, 1 \leq k \leq N+1$, depend on $w$ only, then $D\left(\mu_{0}\right)-\mu_{0}$ is a function of $w$, and therefore $D\left(\mu_{0}\right)-\mu_{0}$ is a constant; we denote it by $d_{0}$.

If $\lambda \neq-1 / r$ for all $r \in \mathbb{N}$, then $p_{k} \neq 0$ for all $k \in \mathbb{N}$, and Eq. (41) fails to be true.

Consider the case when $\lambda=-1 / r$ for some $r \in \mathbb{N}$. Substitution $u_{j}=e^{-\lambda w_{j}}$ transforms vector fields $\left(-1 / \lambda c_{3}\right) Q_{\lambda},(-1 / \lambda) S_{1}$, and $(-1 / \lambda) S_{0}$ into vector fields

$$
\begin{gathered}
Q_{\lambda}^{*}=\sum_{j=-\infty}^{\infty} \frac{\partial}{\partial u_{j}}, \\
S_{1}^{*}=\sum_{j=-\infty}^{\infty}\left\{\widetilde{\rho}_{j}^{*}+\widetilde{b}^{*}\left(u_{j}\right)\right\} u_{j} \frac{\partial}{\partial u_{j}}, \\
S_{0}^{*}=\sum_{j=-\infty}^{\infty} u_{j} \frac{\partial}{\partial u_{j}},
\end{gathered}
$$

where

$$
\widetilde{\rho}_{j}^{*}=\left\{\begin{array}{ll}
\sum_{k=0}^{j-1}\left(u_{k}^{r}-\widetilde{c}\right) & \text { if } j \geq 1 \\
0 & \text { if } j=0, \\
-\sum_{k=j}^{-1}\left(u_{k}^{r}-\widetilde{c}\right) & \text { if } j \leq-1 .
\end{array} \quad \widetilde{b}^{*}\left(u_{j}\right)=\widetilde{b}\left(r \ln u_{j}\right)\right.
$$

First consider the case $r=1$. We have 


$$
\begin{gathered}
T:=\left[Q_{\lambda}^{*}, S_{1}^{*}\right]=\sum_{j=-\infty}^{\infty}\left\{j u_{j}+\widetilde{\rho}_{j}^{*}+\widetilde{b}^{*}\left(u_{j}\right)+u_{j} \tilde{b}^{* \prime}\left(u_{j}\right)\right\} \frac{\partial}{\partial u_{j}}, \\
K:=\frac{1}{2}\left[Q_{\lambda}^{*}, T\right]=\sum_{j=-\infty}^{\infty}\left\{j+c\left(u_{j}\right)\right\} \frac{\partial}{\partial u_{j}},
\end{gathered}
$$

where $c\left(u_{j}\right)=\widetilde{b}^{* \prime}\left(u_{j}\right)+\frac{1}{2} u_{j} \widetilde{b}^{* \prime \prime}\left(u_{j}\right)$

$$
\begin{gathered}
T_{1}=[T, K]=\gamma_{1} \sum_{j=-\infty}^{\infty}\left\{j^{2}+j g_{1,1}^{(j)}\left(u_{j}\right)+g_{1,0}^{(j)}\left(u, u_{1}, \ldots, u_{j}\right)\right\} \frac{\partial}{\partial u_{j}}, \\
T_{2}=\left[T, T_{1}\right]=\gamma_{2} \sum_{j=-\infty}^{\infty}\left\{j^{3}+j^{2} g_{2,2}^{(j)}\left(u_{j}\right)+j g_{2,1}^{(j)}\left(u, u_{1}, \ldots, u_{j}\right)+g_{2,0}^{(j)}\left(u, u_{1}, \ldots, u_{j}\right)\right\} \frac{\partial}{\partial u_{j}},
\end{gathered}
$$

where $\gamma_{1}=-\frac{3}{2}$ and $\gamma_{2} \neq 0$.

Construct vector fields, $T_{n}=\left[T, T_{n-1}\right], n \geq 3$. Direct calculations show that

$$
T_{n}=\gamma_{n} \sum_{j=0}^{\infty}\left\{j^{n+1}+j^{n} g_{n, n}\left(u_{j}\right)+\sum_{k=0}^{n-1} j^{k} g_{n, k}\left(u, u_{1}, \ldots, u_{j}\right)\right\} \frac{\partial}{\partial u_{j}}+\sum_{j=-\infty}^{-1} a_{j} \frac{\partial}{\partial u_{j}}, \quad n \geq 1 .
$$

Since $\left\{T_{n}\right\}_{n=1}^{\infty}$ is an infinite sequence of linearly independent vector fields from $L_{x}$, then case $r$ $=1$ fails to exist.

Consider case $r \geq 2$. We have

$$
a d_{Q_{\lambda}^{*}} S_{1}^{*}=\left[Q_{\lambda}^{*}, S_{1}^{*}\right]=\sum_{j=-\infty}^{\infty}\left\{\operatorname{sgn}(j) r\left(\sum_{k=0}^{j-1} u_{k}^{r-1}\right) u_{j}+\widetilde{\rho}_{j}^{*}+\tilde{b}^{*}\left(u_{j}\right)+u_{j} \widetilde{b}^{* \prime}\left(u_{j}\right)\right\} \frac{\partial}{\partial u_{j}}
$$

and

$$
a d_{Q_{\lambda}^{*}}^{r} S_{1}^{*}=\sum_{j=-\infty}^{\infty}\left\{r ! j u_{j}+\operatorname{sgn}(j) r ! \sum_{k=0}^{j-1} u_{k}+d\left(u_{j}\right)\right\}
$$

for some function $d$,

$$
a d_{Q_{\lambda}^{*}}^{r+1} S_{1}^{*}=\sum_{j=-\infty}^{\infty}\left\{2 r ! j+d^{\prime}\left(u_{j}\right)\right\} \frac{\partial}{\partial u_{j}} .
$$

Note that vector fields $a d_{Q_{\lambda}^{*}}^{r} S_{1}^{*}$ and $a d_{Q_{\lambda}^{*}}^{r+1} S_{1}^{*}$ have coefficients of the same kind as vector fields $T$ and $K$ (from case $r=1$ ) have. It means that $a d_{Q_{\lambda}^{*}}^{r} S_{1}^{*}$ and $a d_{Q_{\lambda}^{*}}^{r+1} S_{1}^{*}$ generate an infinite dimensional Lie algebra. This contradiction implies that case $r \geq 2$ also fails to exist.

Thus, $c_{3}=0$. By interchanging $\lambda$ with $-\lambda$, we obtain that $c_{4}=0$ also. Hence $c_{3}=c_{4}=0$ and $q(w)=c_{2}$.

We already know that $a(\tau)=c_{0} \tau+c_{1}$. The next lemma shows that $c_{0} \neq 0$.

Lemma 8: $c_{0}$ is a nonzero constant.

Proof: Assume the contrary. Then $a(\tau)=c_{1}$ and $c_{1} \neq 0$. Vector fields (37) and (38) become

$$
T_{1}=c_{1} \sum_{j=-\infty}^{\infty} \frac{\partial}{\partial \tau_{j}}=c_{1} \widetilde{T}_{1}
$$

and 


$$
T=-t T_{1}-c_{1} \sum_{j=-\infty}^{\infty}\left\{\rho_{j}+\frac{1}{m c_{1}} b\left(\tau_{j}\right)\right\} \frac{\partial}{\partial \tau_{j}}=-c_{1} t \widetilde{T}_{1}-c_{1} \widetilde{T}
$$

where

$$
\widetilde{T}_{1}=\sum_{j=-\infty}^{\infty} \frac{\partial}{\partial \tau_{j}}, \quad \widetilde{T}=\sum_{j=-\infty}^{\infty}\left\{\rho_{j}+\frac{1}{m c_{1}} b\left(\tau_{j}\right)\right\} \frac{\partial}{\partial \tau_{j}} .
$$

Since

$$
\left[\widetilde{T}_{1},\left[\widetilde{T}_{1}, \widetilde{T}\right]\right]=\frac{1}{m c_{1}} \sum_{j=-\infty}^{\infty} b^{\prime \prime}\left(\tau_{j}\right) \frac{\partial}{\partial \tau_{j}}
$$

and $\widetilde{T}_{1}$ both belong to a finite dimensional $L_{x}$, then, by Lemma 6, (1) $b^{\prime \prime}(\tau)=\widetilde{C}_{1}+\widetilde{C}_{2} e^{\lambda \tau}+\widetilde{C}_{3} e^{-\lambda \tau}$ or (2) $b^{\prime \prime}(\tau)=\widetilde{C}_{1}+\widetilde{C}_{2} \tau+\widetilde{C}_{3} \tau^{2}$ for some constants $\widetilde{C}_{1}-\widetilde{C}_{3}$.

In case (1), $b(\tau)=C_{1}+C_{2} e^{\lambda \tau}+C_{3} e^{-\lambda \tau}+C_{4} \tau^{2}+C_{5} \tau$ and

$$
\left[\widetilde{T}_{1},\left[\widetilde{T}_{1}, \widetilde{T}\right]\right]-\lambda^{2} \widetilde{T}-\frac{2 C_{4}-\lambda^{2} C_{1}}{m c_{1}} \widetilde{T}_{1}=-\lambda^{2} \sum_{j=-\infty}^{\infty}\left\{\rho_{j}+\frac{C_{4} \tau_{j}^{2}+C_{5} \tau_{j}}{m c_{1}}\right\} \frac{\partial}{\partial \tau_{j}}
$$

is an element in $L_{x}$.

In case (2), $b(\tau)=C_{1}+C_{2} \tau+C_{3} \tau^{2}+C_{4} \tau^{3}+C_{5} \tau^{4}$ and

$$
\widetilde{T}-\frac{C_{1}}{m c_{1}} \widetilde{T}_{1}=\sum_{j=-\infty}^{\infty}\left\{\rho_{j}+\frac{C_{2} \tau_{j}+C_{3} \tau_{j}^{2}+C_{4} \tau_{j}^{3}+C_{5} \tau_{j}^{4}}{m c_{1}}\right\} \frac{\partial}{\partial \tau_{j}}
$$

belongs to $L_{x}$.

To finish the proof of the lemma it is enough to show that vector fields

$$
\widetilde{T}_{2}:=\sum_{j=-\infty}^{\infty}\left\{\rho_{j}+C_{2} \tau_{j}+C_{3} \tau_{j}^{2}+C_{4} \tau_{j}^{3}+C_{5} \tau_{j}^{4}\right\} \frac{\partial}{\partial \tau_{j}}
$$

and

$$
\widetilde{T}_{1}=\sum_{j=-\infty}^{\infty} \frac{\partial}{\partial \tau_{j}}
$$

produce an infinite dimensional Lie algebra $L_{x}$ for any fixed constants $C_{2}-C_{5}$. One can prove it by showing that $L_{x}$ contains vector fields $\sum_{j=-\infty}^{\infty} j^{k}\left(\partial / \partial \tau_{j}\right)$ for all $k=1,2, \ldots$. Note that

$$
\left[\widetilde{T}_{1}, \widetilde{T}_{2}\right]=\sum_{j=-\infty}^{\infty}\left(-j+C_{2}+2 C_{3} \tau_{j}+3 C_{4} \tau_{j}^{2}+4 C_{5} \tau_{j}^{3}\right) \frac{\partial}{\partial \tau_{j}} .
$$

There are four cases: (a) $C_{5} \neq 0$, (b) $C_{5}=0, C_{4} \neq 0$, (c) $C_{5}=C_{4}=0, C_{3} \neq 0$, and (d) $C_{5}=C_{4}=C_{3}$ $=0$.

In case (a),

$$
\left[\widetilde{T}_{1},\left[\widetilde{T}_{1},\left[\widetilde{T}_{1}, \widetilde{T}_{2}\right]\right]\right]-6 C_{4} \widetilde{T}_{1}=\sum_{j=-\infty}^{\infty} 24 C_{5} \tau_{j} \frac{\partial}{\partial \tau_{j}}=24 C_{5} P_{1} \in L_{x}, \quad P_{1}=\sum_{j=-\infty}^{\infty} \tau_{j} \frac{\partial}{\partial \tau_{j}},
$$




$$
\left[\widetilde{T}_{1},\left[\widetilde{T}_{1}, \widetilde{T}_{2}\right]\right]=\sum_{j=-\infty}^{\infty}\left\{2 C_{3}+6 C_{4} \tau_{j}+12 C_{5} \tau_{j}^{2}\right\} \frac{\partial}{\partial \tau_{j}} \in L_{x},
$$

and therefore,

$$
P_{2}:=\sum_{j=-\infty}^{\infty} \tau_{j}^{2} \frac{\partial}{\partial \tau_{j}} \in L_{x}
$$

and

$$
\widetilde{T}_{3}:=\left[\widetilde{T}_{1}, \widetilde{T}_{2}\right]-C_{2} \widetilde{T}_{1}-2 C_{3} P_{1}-3 C_{4} P_{2}=\sum_{j=-\infty}^{\infty}\left(-j+4 C_{5} \tau_{j}^{3}\right) \frac{\partial}{\partial \tau_{j}} \in L_{x} .
$$

We have

$$
J_{1}:=-\frac{1}{3}\left(\left[\widetilde{T}_{3}, P_{1}\right]+2 \widetilde{T}_{3}\right)=\sum_{j=-\infty}^{\infty} j \frac{\partial}{\partial \tau_{j}} \in L_{x} .
$$

Now,

$$
\left[J_{1},\left[J_{1}, P_{2}\right]\right]=\frac{1}{2} \sum_{j=-\infty}^{\infty} j^{2} \frac{\partial}{\partial \tau_{j}} \in L_{x} .
$$

Assuming $J_{k}=\sum_{j=-\infty}^{\infty} j^{k}\left(\partial / \partial \tau_{j}\right) \in L_{x}$ we have that

$$
J_{k+1}:=\frac{1}{2}\left[J_{1},\left[J_{k}, P_{2}\right]\right]=\sum_{j=-\infty}^{\infty} j^{k+1} \frac{\partial}{\partial \tau_{j}} \in L_{x} .
$$

In case (b) we have

$$
P_{1}:=\frac{1}{6 C_{4}}\left\{\left[\widetilde{T}_{1},\left[\widetilde{T}_{1}, \widetilde{T}_{2}\right]\right]-2 C_{3} T_{1}\right\}=\sum_{j=-\infty}^{\infty} \tau_{j} \frac{\partial}{\partial \tau_{j}} \in L_{x}
$$

and

$$
\widetilde{T}_{3}=\left[\widetilde{T}_{1}, \widetilde{T}_{2}\right]-C_{2} \widetilde{T}_{1}-2 C_{3} P_{1}=\sum_{j=-\infty}^{\infty}\left(-j+3 C_{4} \tau_{j}^{2}\right) \frac{\partial}{\partial \tau_{j}} \in L_{x} .
$$

We have

$$
J_{1}:=-\frac{1}{2}\left(\left[\widetilde{T}_{3}, P_{1}\right]+\widetilde{T}_{3}\right)=\sum_{j=-\infty}^{\infty} j \frac{\partial}{\partial \tau_{j}} \in L_{x}
$$

and

$$
P_{2}=\frac{1}{6 C_{4}}\left(\widetilde{T}_{3}-\left[\widetilde{T}_{3}, P_{1}\right]\right)=\sum_{j=-\infty}^{\infty} \tau_{j}^{2} \frac{\partial}{\partial \tau_{j}} \in L_{x} .
$$

As it was shown in the proof of case (a), $J_{1}$ and $P_{2}$ produce an infinite dimensional Lie algebra.

In case (c),

$$
\widetilde{T}_{3}=\left[\widetilde{T}_{1}, \widetilde{T}_{2}\right]-C_{2} \widetilde{T}_{1}=\sum_{j=-\infty}^{\infty}\left(-j+2 C_{3} \tau_{j}\right) \frac{\partial}{\partial \tau_{j}} \in L_{x}
$$




$$
\widetilde{T}_{4}=\left[\widetilde{T}_{3}, \widetilde{T}_{2}\right]=\sum_{j=-\infty}^{\infty}\left(\frac{j(j-1)}{2}-j C_{2}-2 C_{3} j \tau_{j}+2 C_{3}^{2} \tau_{j}^{2}\right) \frac{\partial}{\partial \tau_{j}} \in L_{x}
$$

Also,

$$
\tilde{T}_{5}=\left[\widetilde{T}_{3}, \widetilde{T}_{4}\right]=2 C_{3} \sum_{j=-\infty}^{\infty}\left(\frac{j(j+1)}{2}+C_{2} j-2 C_{3} j \tau_{j}+2 C_{3}^{2} \tau_{j}^{2}\right) \frac{\partial}{\partial \tau_{j}} \in L_{x} .
$$

Since $\widetilde{T}_{4}$ and $\widetilde{T}_{5}$ both belong to $L_{x}$, then either

(i)

$$
J_{1}=\sum_{j=-\infty}^{\infty} j \frac{\partial}{\partial \tau_{j}} \in L_{x}, \quad \tilde{T}_{6}=\sum_{j=-\infty}^{\infty}\left(\frac{j^{2}}{2}-2 C_{3} j \tau_{j}+2 C_{3}^{2} \tau_{j}^{2}\right) \frac{\partial}{\partial \tau_{j}} \in L_{x}
$$

or

(ii)

$$
C_{2}=-\frac{1}{2}, \quad \tilde{T}_{6}=\sum_{j=-\infty}^{\infty}\left(\frac{j^{2}}{2}-2 C_{3} j \tau_{j}+2 C_{3}^{2} \tau_{j}^{2}\right) \frac{\partial}{\partial \tau_{j}} \in L_{x}
$$

In case (c) (i),

$$
P_{1}=\frac{1}{4 C_{3}^{2}}\left\{\left[\widetilde{T}_{1}, \widetilde{T}_{6}\right]+2 C_{3} J_{1}\right\}=\sum_{j=-\infty}^{\infty} \tau_{j} \frac{\partial}{\partial \tau_{j}} \in L_{x} .
$$

Since

$$
\left[P_{1}, \widetilde{T}_{6}\right]=\sum_{j=-\infty}^{\infty}\left(-\frac{j^{2}}{2}+2 C_{3}^{2} \tau_{j}^{2}\right) \frac{\partial}{\partial \tau_{j}}
$$

and

$$
\left[P_{1},\left[P_{1}, \tilde{T}_{6}\right]\right]=\sum_{j=-\infty}^{\infty}\left(\frac{j^{2}}{2}+2 C_{3}^{2} \tau_{j}^{2}\right) \frac{\partial}{\partial \tau_{j}}
$$

both belong to $L_{x}$, then

$$
J_{2}=\sum_{j=-\infty}^{\infty} j^{2} \frac{\partial}{\partial \tau_{j}} \in L_{x}, \quad P_{2}=\sum_{j=-\infty}^{\infty} \tau_{j}^{2} \frac{\partial}{\partial \tau_{j}} \in L_{x} .
$$

$P_{2}$ and $J_{1}$ generate an infinite dimensional Lie algebra.

In case (c) (ii),

$$
\widetilde{T}_{1}=\sum_{j=-\infty}^{\infty} \frac{\partial}{\partial \tau_{j}}, \quad \widetilde{T}_{2}=\sum_{j=-\infty}^{\infty}\left(C_{3} \tau_{j}^{2}-\frac{1}{2} \tau_{j}+\rho_{j}\right) \frac{\partial}{\partial \tau_{j}}
$$

Note that the Lie algebra generated by the vector fields

$$
\widetilde{T}_{2}^{*}=\widetilde{T}_{2}-\left(C_{3} \tau^{2}-\frac{1}{2} \tau\right) \widetilde{T}_{1}=d\left(\tau, \tau_{1}\right) \frac{\partial}{\partial \tau_{1}}-d\left(\tau_{-1}, \tau\right) \frac{\partial}{\partial \tau_{-1}}+\left(d\left(\tau, \tau_{1}\right)+d\left(\tau_{1}, \tau_{2}\right)\right) \frac{\partial}{\partial \tau_{2}}+\cdots
$$

and 


$$
\widetilde{T}_{1}=\sum_{j=-\infty}^{\infty} \frac{\partial}{\partial \tau_{j}}
$$

is infinite dimensional. It can be proved by comparing this algebra with the infinite dimensional characteristic Lie algebra of the chain

$$
t_{1 x}=t_{x}+C_{3}\left(t_{1}^{2}-t^{2}\right)-\frac{1}{2}\left(t_{1}+t\right) .
$$

Indeed, the Lie algebra $L_{x 1}$ for (42) is generated by operators (18) and (19) with $d\left(t, t_{1}\right)=C_{3}\left(t_{1}^{2}\right.$ $\left.-t^{2}\right)-\frac{1}{2}\left(t_{1}+t\right)$. To keep standard notations we set $a(\tau)=-2 C_{3} \tau-1$ and $b(\tau)=C_{3} \tau^{2}+\frac{1}{2} \tau$. Note that since $C_{3} \neq 0$, function $a(\tau)$ is not a constant. It follows from Theorem 3 proved below that the characteristic Lie algebras $L_{x}$ (and therefore algebra $L_{x 1}$ ) for Eq. (42) is of infinite dimension. Thus, in case (c) (ii) we also have an infinite dimensional Lie algebra $L_{x}$.

In case $(d)$,

$$
\widetilde{T}_{2}=\sum_{j=-\infty}^{\infty}\left(-\tau-\tau_{1}-\cdots-\tau_{j-1}+C_{2} \tau_{j}\right) \frac{\partial}{\partial \tau_{j}} \in L_{x}
$$

Then

$$
J_{1}=c_{2} \widetilde{T}_{1}-\left[\widetilde{T}_{1}, \widetilde{T}_{2}\right]=\sum_{j=-\infty}^{\infty} j \frac{\partial}{\partial \tau_{j}} \in L_{x}
$$

and

$$
J_{2}=-2\left(\left[J_{1}, \widetilde{T}_{2}\right]-\left(\frac{1}{2}+C_{2}\right) J_{1}\right)=\sum_{j=-\infty}^{\infty} j^{2} \frac{\partial}{\partial \tau_{j}} \in L_{x} .
$$

Assuming that $J_{k}, 1 \leq k \leq n$, belong to $L_{x}$, by considering $\left[J_{n}, \widetilde{T}_{2}\right]$ one may show that $J_{n+1}$ $=\Sigma_{j=-\infty}^{\infty} j^{k+1}\left(\partial / \partial \tau_{j}\right) \in L_{x}$. It implies that $L_{x}$ is of infinite dimension.

Let us introduce new variables,

$$
w_{j}=\ln \left(\tau_{j}+\frac{c_{1}}{c_{0}}\right) .
$$

Vector fields $T_{1}$ and $T$ in variables $w_{j}$ can be rewritten as

$$
\begin{gathered}
T_{1}=c_{0} \sum_{j=-\infty}^{\infty} \frac{\partial}{\partial w_{j}}=c_{0} S_{0}, \\
T=-t c_{0} S_{0}+c_{0} \sum_{j=-\infty}^{\infty}\left\{\widetilde{\rho}_{j}+\widetilde{b}\left(w_{j}\right)\right\} \frac{\partial}{\partial w_{j}}=-c_{0} t S_{0}+c_{0} S_{1},
\end{gathered}
$$

where

$$
S_{0}=\sum_{j=-\infty}^{\infty} \frac{\partial}{\partial w_{j}}, \quad S_{1}=\sum_{j=-\infty}^{\infty}\left\{\widetilde{\boldsymbol{\rho}}_{j}+\widetilde{b}\left(w_{j}\right)\right\} \frac{\partial}{\partial w_{j}},
$$




$$
\tilde{\rho}_{j}= \begin{cases}\sum_{k=0}^{j-1}\left(e^{w_{k}}-\tilde{c}\right) & \text { if } j \geq 1 \\ 0 & \text { if } j=0, \quad \tilde{c}=\frac{c_{1}}{c_{0}}, \quad \tilde{b}\left(w_{j}\right)=-\frac{1}{m}\left(\frac{b\left(\tau_{j}\right)}{c_{0} \tau_{j}+c_{1}}\right) \\ -\sum_{k=j}\left(e^{w_{k}}-\tilde{c}\right) & \text { if } j \leq-1 .\end{cases}
$$

We have

$$
D S_{0} D^{-1}=S_{0}, \quad D S_{1} D^{-1}=S_{1}-\left(e^{w}-\widetilde{c}\right) S_{0} .
$$

These lemmas allow one to prove the following theorem.

Theorem 4: If the equation

$$
t_{1 x}=t_{x}+a(\tau) t^{m}+b(\tau) t^{m-1}+\cdots, \quad m \geq 1,
$$

admits a nontrivial $x$-integral, then

(1) $a(\tau)=c_{0} \tau$ and $b(\tau)=c_{2} \tau^{2}+c_{3} \tau$, where $c_{0}, c_{2}$, and $c_{3}$ are some constants.

(2) $m=1$.

Proof: Consider case (1). Define vector field

$$
Q=\left[S_{0},\left[S_{0}, S_{1}\right]\right]-\left[S_{0}, S_{1}\right]=\sum_{j=-\infty}^{\infty}\left(\widetilde{b^{\prime \prime}}\left(w_{j}\right)-{\tilde{b^{\prime}}}^{\prime}\left(w_{j}\right)\right) \frac{\partial}{\partial w_{j}}
$$

By Lemma $7, \tilde{b}^{\prime \prime}(w)-\tilde{b}^{\prime}(w)=C$ for some constant $C$. Thus, $\tilde{b}(w)=C_{0}+C_{1} e^{w}+C_{2} w$ for some constants $C_{1}, C_{2}$, and $C_{0}$. Consider vector fields

$$
\begin{gathered}
P=\left(C_{2}-C_{0}\right) S_{0}+S_{1}-\left[S_{0}, S_{1}\right]=\sum_{j=-\infty}^{\infty}\left(C_{2} w_{j}+\tilde{c} j\right) \frac{\partial}{\partial w_{j}}, \\
R=\left[S_{0},\left[S_{0}, S_{1}\right]\right]=\sum_{j=1}^{\infty}\left\{\left(\sum_{k=1}^{j} e^{w_{k}}\right)+C_{1} e^{w_{j}}\right\} \frac{\partial}{\partial w_{j}}+C_{1} e^{w} \frac{\partial}{\partial w}-\sum_{j=-\infty}^{-1}\left\{\left(\sum_{k=j}^{-1} e^{w_{k}}\right)+C_{1} e^{w_{j}}\right\} \frac{\partial}{\partial w_{j}}, \\
R_{1}=[P, R], \quad R_{n+1}=\left[P, R_{n}\right], \quad n \geq 1 .
\end{gathered}
$$

Then

$$
\begin{aligned}
R_{n}= & \sum_{j \geq 0}\left\{e^{w_{j}}\left(C_{1} C_{2}^{n} w_{j}^{n}+P_{n, j}\right)+r_{n, j}\left(w, w_{1}, \ldots, w_{j-1}\right)\right\} \frac{\partial}{\partial w_{j}} \\
& +\sum_{j \leq-1}\left\{e^{w_{j}}\left(\left(C_{1}-1\right) C_{2}^{n} w_{j}^{n}+P_{n, j}\right)+r_{n, j}\left(w_{-1}, w_{-2}, \ldots, w_{j+1}\right)\right\} \frac{\partial}{\partial w_{j}}
\end{aligned}
$$

where $P_{n, j}=P_{n, j}\left(w_{j}, j\right)$ is a polynomial of degree $n-1$ whose coefficients depend on $j ; r_{n, j}$ are the functions that do not depend on $w_{j}$. Since all vector fields $R_{n}$ belong to a finite dimensional Lie algebra $L_{x}$, then $C_{1} C_{2}=\left(C_{1}-1\right) C_{2}=0$, or the same, $C_{2}=0$. Therefore,

$$
\tilde{b}(w)=C_{0}+C_{1} e^{w}
$$

Since $C_{2}=0$, then 


$$
\begin{gathered}
P=\widetilde{c} \sum_{j=-\infty}^{\infty} j \frac{\partial}{\partial w_{j}}, \\
R=\sum_{j=1}^{\infty}\left\{\left(\sum_{k=1}^{j} e^{w_{k}}\right)+C_{1} e^{w_{j}}\right\} \frac{\partial}{\partial w_{j}}+C_{1} e^{w} \frac{\partial}{\partial w}-\sum_{j=-\infty}^{-1}\left\{\left(\sum_{k=j}^{-1} e^{w_{k}}\right)+C_{1} e^{w_{j}}\right\} \frac{\partial}{\partial w_{j}}
\end{gathered}
$$

and

$$
\begin{aligned}
R_{n}= & \widetilde{c}^{n} \sum_{j=1}^{\infty}\left\{e^{w_{1}}+2^{n} e^{w_{2}}+(j-1)^{n} e^{w_{j-1}}+j^{n} C_{1} e^{w_{j}}\right\} \frac{\partial}{\partial w_{j}} \\
& -\widetilde{c}^{n} \sum_{j=-\infty}^{-1}\left\{(-1)^{n} e^{w_{-1}}+(-2)^{n} e^{w_{-2}}+(j)^{n} e^{w_{j}}+j^{n} C_{1} e^{w_{j}}\right\} \frac{\partial}{\partial w_{j}} .
\end{aligned}
$$

Again, vector fields $R_{n}$ belong to a finite dimensional Lie algebra only if $\tilde{c}=0$, or the same, $c_{1}$ $=0$. It implies that

$$
a(\tau)=c_{0} \tau, \quad b(\tau)=c_{2} \tau^{2}+c_{3} \tau
$$

Consider case (2). Assume the contrary, that is, $m \geq 2$. Then the following vector field:

$$
\begin{aligned}
\frac{1}{m !} a d_{\tilde{X}}^{m-2}(\tilde{Y})= & Y_{(1 / 2) a(\tau) t^{2}+(1 / m) b(\tau) t+\{1 /[m(m-1)]\} c(\tau)}=-\sum_{j=-\infty}^{\infty}\left(\frac{1}{2} a\left(\tau_{j}\right) t_{j}^{2}+\frac{1}{m} b\left(\tau_{j}\right) t_{j}+\frac{1}{m(m-1)} c\left(\tau_{j}\right)\right) \frac{\partial}{\partial \tau_{j}} \\
= & -\sum_{j=-\infty}^{\infty}\left(\frac{1}{2} a\left(\tau_{j}\right)\left(t+\rho_{j}\right)^{2}+\frac{1}{m} b\left(\tau_{j}\right)\left(t+\rho_{j}\right)+\frac{1}{m(m-1)} c\left(\tau_{j}\right)\right) \frac{\partial}{\partial \tau_{j}}-\frac{t^{2}}{2} \sum_{j=-\infty}^{\infty} a\left(\tau_{j}\right) \frac{\partial}{\partial \tau_{j}} \\
& -t \sum_{j=-\infty}^{\infty}\left\{a\left(\tau_{j}\right) \rho_{j}+\frac{1}{m} b\left(\tau_{j}\right)\right\} \frac{\partial}{\partial \tau_{j}}-\sum_{j=-\infty}^{\infty}\left\{\frac{1}{2} a\left(\tau_{j}\right) \rho_{j}^{2}+\frac{1}{m} b\left(\tau_{j}\right)+\frac{1}{m(m-1)} c\left(\tau_{j}\right)\right\} \frac{\partial}{\partial \tau_{j}},
\end{aligned}
$$

is in $L_{x}$. In variables $w_{j}=\ln \tau_{j}$,

$$
\frac{1}{m !} a d_{\tilde{X}}^{m-2}(\tilde{Y})=-\frac{t^{2}}{2} c_{0} S_{0}+t c_{0} S_{1}-c_{0} S_{2}
$$

where

$$
S_{2}=\sum_{j=-\infty}^{\infty}\left\{\frac{1}{2} \widetilde{\rho}_{j}^{2}-\tilde{b}\left(w_{j}\right) \widetilde{\rho}_{j}+\widetilde{c}\left(w_{j}\right)\right\} \frac{\partial}{\partial w_{j}}, \quad \widetilde{c}\left(w_{j}\right)=\frac{c\left(\tau_{j}\right)}{m(m-1) \tau_{j}} .
$$

The vector fields $S_{0}$ and $S_{1}$ are as in Lemma 7 . We have

$$
\left[S_{0}, S_{2}\right]=2 S_{2}+C_{0} S_{1}+P, \quad P=\sum_{j=-\infty}^{\infty} r\left(w_{j}\right) \frac{\partial}{\partial w_{j}}, \quad r(w)=\tilde{c}^{\prime}(w)-2 \widetilde{c}(w)-C_{0} \tilde{b}(w) .
$$

Construct the sequence

$$
S_{3}=\left[S_{1}, S_{2}\right], \quad S_{n+1}=\left[S_{1}, S_{n}\right], \quad n \geq 2 .
$$

One can prove by induction on $n$ that 


$$
\left[S_{0}, S_{n}\right]=n S_{n}+\sum_{k=0}^{n-1} \nu_{n, k} S_{k}
$$

and

$$
D S_{n} D^{-1}=S_{n}+\left\{\frac{n(n-1)}{2}-1\right\} e^{w} S_{n-1}+\sum_{k=0}^{n-2} \eta(n, k) S_{k}, \quad n \geq 3 .
$$

Since $L_{x}$ is of finite dimension, then there exists a natural number $N$ such that

$$
S_{N+1}=\mu_{N} S_{N}+\mu_{N-1} S_{N-1}+\cdots+\mu_{0} S_{0} .
$$

Then

$$
D S_{N+1} D^{-1}=D\left(\mu_{N}\right) D S_{N} D^{-1}+D\left(\mu_{N-1}\right) D S_{N-1} D^{-1}+\cdots+D\left(\mu_{0}\right) D S_{0} D^{-1} .
$$

On the other hand,

$$
D S_{N+1} D^{-1}=S_{N+1}+\left\{\frac{(N+1) N}{2}-1\right\} e^{w} S_{N}+\cdots .
$$

We compare the coefficients before $S_{N}$ and have two equations:

$$
D\left(\mu_{N}\right)=\mu_{N}+\left\{\frac{(N+1) N}{2}-1\right\} e^{w}, \quad N \geq 2,
$$

and

$$
D\left(\mu_{1}\right)=\mu_{1}+e^{w}, \quad N=1 .
$$

Both equations are contradictory. Therefore, our assumption that $m \geq 2$ was wrong.

\section{NONZERO ROOT}

Lemma 9: Assume that Eq. (12) admits a nontrivial $x$-integral. Then the characteristic polynomial of Eq. (25) can only have simple nonzero roots.

Proof: Assume that $m_{1} \geq 2$. Introduce polynomials

$$
\Lambda_{\alpha_{1}}^{(2)}(\lambda)=\frac{\Lambda(\lambda)}{\left(\lambda-\alpha_{1}\right)^{2}}, \quad \Lambda_{\alpha_{1}}(\lambda)=\frac{\Lambda(\lambda)}{\left(\lambda-\alpha_{1}\right)} .
$$

Consider vector fields

$$
\begin{gathered}
S_{0}^{*}=\Lambda_{\alpha_{1}}^{(2)}\left(a d_{\tilde{X}}\right) Y_{d}=Y_{A(\tau) e^{\alpha_{1} t}}, \\
S_{1}^{*}=\Lambda_{\alpha_{1}}\left(a d_{\tilde{X}}\right) Y_{d}=Y_{(A(\tau) t+B(\tau)) e^{\alpha_{1} t}}
\end{gathered}
$$

from the Lie algebra $L_{x}$.

In variables $\tau_{j}=t_{j}-t_{j+1}$, vector fields $S_{0}^{*}$ and $S_{1}^{*}$ become

$$
S_{0}^{*}=-e^{\alpha_{1} t} \sum_{j=-\infty}^{\infty} A\left(\tau_{j}\right) e^{\alpha_{1} \rho_{j}} \frac{\partial}{\partial \tau_{j}}=-e^{\alpha_{1} t} S_{0},
$$




$$
S_{1}^{*}=-t e^{\alpha_{1} t} S_{0}-e^{\alpha_{1} t} \sum_{j=-\infty}^{\infty}\left\{A\left(\tau_{j}\right) \rho_{j}+B\left(\tau_{j}\right)\right\} e^{\alpha_{1} \rho_{j}} \frac{\partial}{\partial \tau_{j}}=-t e^{\alpha_{1} t} S_{0}-e^{\alpha_{1} t} S_{1},
$$

with $S_{0}=\sum_{j=-\infty}^{\infty} A\left(\tau_{j}\right) e^{\alpha_{1} \rho_{j}}\left(\partial / \partial \tau_{j}\right)$ and $S_{1}=\sum_{j=-\infty}^{\infty}\left\{A\left(\tau_{j}\right) \rho_{j}+B\left(\tau_{j}\right)\right\} e^{\alpha_{1} \rho_{j}}\left(\partial / \partial \tau_{j}\right)$.

Direct calculations show that

$$
D S_{0} D^{-1}=e^{\alpha_{1} \tau} S_{0}, \quad D S_{1} D^{-1}=e^{\alpha_{1} \tau} S_{1}+\tau e^{\alpha_{1} \tau} S_{0} .
$$

Define the sequence

$$
S_{2}=\left[S_{0}, S_{1}\right], \quad S_{n+1}=\left[S_{0}, S_{n}\right], \quad n \geq 2 .
$$

One can easily show that

$$
D S_{2} D^{-1}=e^{2 \alpha_{1} \tau} S_{2}+\alpha_{1} e^{2 \alpha_{1} \tau} A(\tau) S_{1}+e^{2 \alpha_{1} \tau}\left(A(\tau)-\alpha_{1} B(\tau)\right) S_{0} .
$$

It can be proved by induction on $n$ that

$$
D S_{n} D^{-1}=e^{n \alpha_{1} \tau} S_{n}+\alpha_{1} \frac{n(n-1)}{2} e^{n \alpha_{1} \tau} A(\tau) S_{n-1}+\sum_{k=0}^{n-2} \gamma(n, k) S_{k} .
$$

Since the dimension of $L_{x}$ is finite and $S_{0}, S_{1}, \ldots$ are elements of $L_{x}$, then there exists a natural number $N$ such that

$$
S_{N+1}=\mu_{N} S_{N}+\mu_{N-1} S_{N-1}+\cdots+\mu_{0} S_{0},
$$

and $S_{0}, S_{1}, \ldots, S_{N}$ are linearly independent. Therefore,

$$
D S_{N+1} D^{-1}=D\left(\mu_{N}\right) D S_{N} D^{-1}+D\left(\mu_{N-1}\right) D S_{N-1} D^{-1}+\cdots+D\left(\mu_{0}\right) D S_{0} D^{-1} .
$$

On the other hand,

$$
D S_{N+1} D^{-1}=e^{(N+1) \alpha_{1} \tau} S_{N+1}+\alpha_{1} \frac{(N+1) N}{2} e^{(N+1) \alpha_{1} \tau} A(\tau) S_{N}+\sum_{k=0}^{N-1} \gamma(N+1, k) S_{k} .
$$

By comparing the coefficients before $S_{N}$ in the last two equations we have

$$
e^{(N+1) \alpha_{1} \tau} \mu_{N}+\frac{\alpha_{1}(N+1) N}{2} e^{(N+1) \alpha_{1} \tau} A(\tau)=D\left(\mu_{N}\right) e^{N \alpha_{1} \tau} .
$$

It follows at once that $\mu_{N}$ is a constant and then

$$
A(\tau)=C\left(e^{-\alpha_{1} \tau}-1\right), \quad C=\frac{2 \mu_{N}}{\alpha_{1} N(N+1)} .
$$

Let us construct a new infinite sequence of vector fields belonging to $L_{x}$, enumerated by a multiindex,

$$
\begin{gathered}
T_{0}:=S_{1}, \quad T_{1}:=S_{0}, \quad T_{2}=\left[S_{1}, T_{1}\right], \quad T_{n+1}=\left[S_{1}, T_{n}\right], \quad n \geq 2, \quad T_{n, 0}=\left[S_{0}, T_{n}\right], \\
T_{n, 0, i_{1}, \ldots, i_{n-1}, i_{n}}=\left[S_{i_{n}}, T_{n, 0, i_{1}, \ldots, i_{n-1}}\right], \quad i_{j} \in\{0 ; 1\} .
\end{gathered}
$$

Direct calculations show that

$$
D T_{2} D^{-1}=e^{2 \alpha_{1} \tau} T_{2}+e^{2 \alpha_{1} \tau}\left(\alpha_{1} B-A\right) T_{1}-\alpha_{1} e^{2 \alpha_{1} \tau} A T_{0},
$$




$$
D T_{3} D^{-1}=e^{3 \alpha_{1} \tau} T_{3}+e^{3 \alpha_{1} \tau}\left(3 \alpha_{1} B-A+3 \alpha_{1} \tau A\right) T_{2}+\tau e^{3 \alpha_{1} \tau} T_{2,0}+\sum_{m(\beta)<2} \nu(3, \beta) T_{\beta}
$$

Here and below we use functions $m=m(\beta)$ and $l=l(\beta)$ defined in Sec. III. It can be proved by induction on $n$ that

$$
D T_{n} D^{-1}=e^{n \alpha_{1} \tau} T_{n}+e^{n \alpha_{1} \tau}\left\{c_{n} B-A+c_{n} \tau A\right\} T_{n-1}+\tau e^{n \alpha_{1} \tau} \sum_{m(\beta)=n-1, l(\beta)=1} \nu^{*}(n, \beta) T_{\beta}+\sum_{m(\beta) \leq n-2} \nu(n, \beta) T_{\beta},
$$

where

$$
c_{n}=\frac{\alpha_{1} n(n-1)}{2}
$$

and $\nu^{*}(n, \beta)$ are constants for any $\beta$ with $m(\beta)=n-1$ and $l(\beta)=1$.

In general, for any $\gamma$,

$$
D T_{\gamma} D^{-1}=e^{(m(\gamma)+l(\gamma)) \alpha_{1} \tau} T_{\gamma}+\sum_{m(\beta) \leq m(\gamma)-1} \nu(\gamma, \beta) T_{\beta} .
$$

Among the vector fields $T_{\beta}$ we choose a system $P$ of linearly independent vector fields in such a way that for some natural number $N$, we have the following:

(i) $T_{k} \in P, k \leq N$.

(ii) $m(\beta) \leq N$ for any $T_{\beta} \in P$.

(iii) For any $T_{\gamma}$ with $m(\gamma) \leq N$ we have $T_{\gamma}=\Sigma_{T_{\beta} \in P, m(\beta) \leq m(\gamma)} \mu(\gamma, \beta) T_{\beta}$. Also

$$
T_{N+1}=\mu(N+1, N) T_{N}+\sum_{T_{\beta} \in P} \mu(N+1, \beta) T_{\beta} .
$$

(iv) For any $T_{\gamma} \notin P$ with $m(\gamma)=N$ and $l(\gamma)=1$, we have $\mu(\gamma, N)=0$.

Indeed,

$$
D T_{\gamma} D^{-1}=D(\mu(\gamma, N)) D T_{N} D^{-1}+\sum_{T_{\beta} \in P, \beta \neq N} D(\mu(\gamma, \beta)) D T_{\beta} D^{-1} .
$$

On the other hand,

$$
\begin{aligned}
D T_{\gamma} D^{-1} & =e^{(m(\gamma)+l(\gamma)) \alpha_{1} \tau} T_{\gamma}+\sum_{m(\beta) \leq N-1} \nu(\gamma, \beta) T_{\beta} \\
& =e^{(N+1) \alpha_{1} \tau}\left\{\mu(\gamma, N) T_{N}+\sum_{T_{\beta} \in P, m(\beta) \leq N, \beta \neq N} \mu(\gamma, \beta) T_{\beta}\right\}+\sum_{m(\beta) \leq N-1} \nu(\gamma, \beta) T_{\beta} .
\end{aligned}
$$

By comparing the coefficients before $T_{N}$ we have

$$
e^{(N+1) \alpha_{1} \tau} \mu(\gamma, N)=D(\mu(\gamma, N)) e^{N \alpha_{1} \tau},
$$

which proves $\mu(\gamma, N)=0$ for any $\gamma$ with $m(\gamma)=N$ and $l(\gamma)=1$. We have

$$
T_{N+1}=\mu_{N} T_{N}+\sum_{T_{\beta} \in P} \mu(N+1, \beta) T_{\beta}
$$

Here $\mu_{N}=\mu(N+1, N)$. Then

$$
D T_{N+1} D^{-1}=D\left(\mu_{N}\right) D T_{N} D^{-1}+\sum_{T_{\beta} \in P} D(\mu(N+1, \beta)) D T_{\beta} D^{-1} .
$$

We continue and have 


$$
\begin{aligned}
e^{(N+1) \alpha_{1} \tau}\left\{\mu_{N} T_{N}+\sum_{T_{\beta} \in P} \mu(N+1, \beta) T_{\beta}\right\}+e^{(N+1) \alpha_{1} \tau\left\{c_{N+1} B-A+c_{N+1} \tau A\right\} T_{N}} \\
+\tau e^{(N+1) \alpha_{1} \tau} \sum_{m(\beta)=N, l(\beta)=1} \nu^{*}(N+1, \beta) T_{\beta}+\sum_{m(\beta) \leq N-1} \nu(N+1, \beta) T_{\beta} \\
=D\left(\mu_{N}\right)\left\{e^{N \alpha_{1} \tau} T_{N}+\sum_{m(\beta) \leq N-1} \nu(N, \beta) T_{\beta}\right\} \\
+\sum_{T_{\beta} \in P} D(\mu(N+1, \beta))\left\{e^{(m(\beta)+l(\beta)) \alpha_{1} \tau} T_{\beta}+\sum_{m(r) \leq N-1} \nu(\beta, r) T_{r}\right\} .
\end{aligned}
$$

We compare the coefficients before $T_{N}$ and get

$$
e^{(N+1) \alpha_{1} \tau} \mu_{N}+e^{(N+1) \alpha_{1} \tau}\left\{c_{N+1} B-A+c_{N+1} \tau A\right\}=e^{N \alpha_{1} \tau} D\left(\mu_{N}\right) .
$$

Note that, by property (iv), we do not have term $\tau e^{(N+1) \alpha_{1} \tau}$ in the left side of the last equality. Thus, using the expression for $A(\tau)=C\left(e^{-\alpha_{1} \tau}-1\right)$ and the fact that $\mu_{N}$ is a constant, we have

$$
B(\tau)=C_{1} A+C_{2} \tau A=C_{1}\left(e^{-\alpha_{1} \tau}-1\right)+C_{2} \tau\left(e^{-\alpha_{1} \tau}-1\right),
$$

where

$$
C_{1}=\frac{\mu_{N}}{C c_{N+1}}+\frac{1}{c_{N+1}}, \quad C_{2}=-1 .
$$

We introduce new vector fields

$$
\begin{gathered}
\tilde{S}_{0}=\frac{1}{C} S_{0}=\left(e^{-\alpha_{1} \tau}-1\right) \frac{\partial}{\partial \tau}+\cdots, \quad \tilde{S}_{1}=\frac{1}{C} S_{1}+\frac{C_{1}}{C} S_{0}=\tau\left(e^{-\alpha_{1} \tau}-1\right) \frac{\partial}{\partial \tau}+\cdots \\
\tilde{S}_{2}=\left[\tilde{S}_{0}, \tilde{S}_{1}\right], \quad \tilde{S}_{n+1}=\left[\tilde{S}_{0}, \tilde{S}_{n}\right], \quad n \geq 2 .
\end{gathered}
$$

We have

$$
\begin{gathered}
D \widetilde{S}_{0} D^{-1}=e^{\alpha_{1} \tau} \widetilde{S}_{0}, \quad D \widetilde{S}_{1} D^{-1}=e^{\alpha_{1} \tau} \widetilde{S}_{1}-\tau e^{\alpha_{1} \tau} \widetilde{S}_{0}, \\
D \widetilde{S}_{n} D^{-1}=\sum_{k=0}^{n} \widetilde{\gamma}(n, k) \widetilde{S}_{k}, \quad \widetilde{\gamma}(n, n)=e^{n \alpha_{1} \tau},
\end{gathered}
$$

where $\widetilde{\gamma}(n, k)$ are functions of $\tau$ only. Since all vector fields $\widetilde{S}_{k}$ belong to a finite dimensional Lie algebra $L_{x}$, then there exists such a natural number $M$ that

$$
\tilde{S}_{M+1}=\tilde{\mu}_{M} \tilde{S}_{M}+\cdots+\tilde{\mu}_{0} \tilde{S}_{0},
$$

and $\widetilde{S}_{0}, \ldots, \widetilde{S}_{M}$ are linearly independent. Then

$$
D \widetilde{S}_{M+1} D^{-1}=D\left(\tilde{\mu}_{M}\right) D \widetilde{S}_{M} D^{-1}+\cdots+D\left(\tilde{\mu}_{0}\right) D \widetilde{S}_{0} D^{-1}
$$

and

$$
\tilde{\gamma}(M+1, M+1)\left\{\tilde{\mu}_{M} \tilde{S}_{M}+\cdots+\tilde{\mu}_{0} \tilde{S}_{0}\right\}+\sum_{k=0}^{M} \tilde{\gamma}(M+1, k) \tilde{S}_{k}=D\left(\tilde{\mu}_{N}\right)\left\{\tilde{\gamma}(M, M) \tilde{S}_{M}+\cdots\right\}+\cdots .
$$

By comparing the coefficients before $\widetilde{S}_{M}$, we have 


$$
e^{(M+1) \alpha_{1} \tau} \widetilde{\mu}_{M}+\widetilde{\gamma}(M+1, M)=D\left(\widetilde{\mu}_{M}\right) e^{M \alpha_{1} \tau},
$$

which implies that $\widetilde{\mu}_{M}$ is a constant. In the same way, by comparing the coefficients before $\widetilde{S}_{M-1}$ and then before $\widetilde{S}_{M-2}$ and so on, one can show that all coefficients $\tilde{\mu}_{k}$ are constants.

One can show by induction on $n$ that for $n \geq 2$,

$$
\tilde{S}_{n}=\left\{\alpha_{1}^{n-2}(-1)^{n-2}(n-2) ! e^{-n \alpha_{1} \tau}+\sum_{k=0}^{n-1} r(n, k) e^{-\alpha_{1} k \tau}\right\} \frac{\partial}{\partial \tau}+\cdots,
$$

where $r(n, k)$ are some constants. Return to equality (43) with constant coefficients $\widetilde{\mu}_{k}$ and compare the coefficients before $\partial / \partial \tau$ :

$$
\begin{aligned}
& \alpha_{1}^{M-1}(-1)^{M-1}(M-1) ! e^{-(M+1) \alpha_{1} \tau}+\sum_{k=0}^{M} r(M+1, k) e^{-\alpha_{1} k \tau}=\tilde{\mu}_{M}\left(\alpha_{1}^{M-2}(-1)^{M-2}(M-2) ! e^{-M \alpha_{1} \tau}\right. \\
& \left.\quad+\sum_{k=0}^{M-1} r(M, k) e^{-\alpha_{1} k \tau}\right)+\cdots+\tilde{\mu}_{0}\left(e^{-\alpha_{1} \tau}-1\right) .
\end{aligned}
$$

The last equality fails to be true. It shows that our assumption that multiplicity $m_{1}$ of a nonzero root $\alpha_{1}$ can be 2 or more was wrong.

If the characteristic polynomial of (25) has only one nonzero root $\alpha$, then $d\left(t, t_{1}\right)=A(t$ $\left.-t_{1}\right) e^{\alpha t}$. In this case Eq. (12) admits a nontrivial $x$-integral (see Sec. I, Theorem 3). In Sec. V we consider a case when the characteristic polynomial of (25) has at least two nonzero roots.

\section{TWO NONZERO ROOTS}

Let $\alpha$ and $\beta$ be two nonzero roots. Consider the vector fields

$$
S_{0}=\sum_{j=-\infty}^{\infty} A\left(\tau_{j}\right) e^{\alpha \rho_{j}} \frac{\partial}{\partial \tau_{j}}, \quad S_{1}=\sum_{j=-\infty}^{\infty} B\left(\tau_{j}\right) e^{\beta \rho_{j}} \frac{\partial}{\partial \tau_{j}}
$$

from the Lie algebra $L_{x}$ and construct a new sequence of vector fields

$$
S_{2}=\left[S_{0}, S_{1}\right], \quad S_{n+1}=\left[S_{0}, S_{n}\right], \quad n \geq 1 .
$$

We have

$$
\begin{gathered}
D S_{0} D^{-1}=e^{\alpha \tau} S_{0}, \quad D S_{1} D^{-1}=e^{\beta \tau} S_{1}, \\
D S_{2} D^{-1}=e^{(\alpha+\beta) \tau} S_{2}+\beta A e^{(\alpha+\beta) \tau} S_{1}-\alpha B e^{(\alpha+\beta) \tau} S_{0} .
\end{gathered}
$$

In general, for any $n \geq 3$,

$$
D S_{n} D^{-1}=e^{((n-1) \alpha+\beta) \tau}\left\{S_{n}+\left(c_{n} \alpha+d_{n} \beta\right) A S_{n-1}+\left(p_{n} A^{\prime}+q_{n} A\right) A S_{n-2}+\sum_{k=0}^{n-2} \nu(n, k) S_{k}\right\},
$$

where

$$
\begin{gathered}
c_{n}=\frac{(n-1)(n-2)}{2}, \quad d_{n}=n-1, \quad p_{n+1}=\frac{n(n-1)}{2}\left\{\frac{n-2}{3} \alpha+\beta\right\}, \quad n \geq 2, \\
q_{n+1}=\frac{n(n-2)(n-1)(3 n-1)}{24} \alpha^{2}+\frac{(n-1)^{2} n}{2} \alpha \beta+\frac{n(n-1)}{2} \beta^{2}, \quad n \geq 2 .
\end{gathered}
$$

Let us consider a particular case when 


$$
S_{2}=\mu_{0} S_{0}+\mu_{1} S_{1}
$$

We have

$$
\begin{aligned}
D S_{2} D^{-1} & =D\left(\mu_{0}\right) e^{\alpha \tau} S_{0}+D\left(\mu_{1}\right) e^{\beta \tau} S_{1}=e^{(\alpha+\beta) \tau} S_{2}+\beta A e^{(\alpha+\beta) \tau} S_{1}-\alpha B e^{(\alpha+\beta) \tau} S_{0} \\
& =e^{(\alpha+\beta) \tau}\left\{\mu_{0} S_{0}+\mu_{1} S_{1}\right\}+\beta A e^{(\alpha+\beta) \tau} S_{1}-\alpha B e^{(\alpha+\beta) \tau} S_{0} .
\end{aligned}
$$

Comparing coefficients before $S_{0}$ and $S_{1}$ produces the following two equations:

$$
e^{(\alpha+\beta) \tau} \mu_{0}-\alpha B e^{(\alpha+\beta) \tau}=D\left(\mu_{0}\right) e^{\alpha \tau}, \quad e^{(\alpha+\beta) \tau} \mu_{1}+\beta A e^{(\alpha+\beta) \tau}=D\left(\mu_{1}\right) e^{\beta \tau} .
$$

It follows that $\mu_{0}$ and $\mu_{1}$ are constants and

$$
B(\tau)=-\frac{\mu_{0}}{\alpha}\left(e^{-\beta \tau}-1\right), \quad A(\tau)=\frac{\mu_{1}}{\beta}\left(e^{-\alpha \tau}-1\right) .
$$

Finally, comparing coefficients before $\partial / \partial \tau$ in Eq. (44) implies that $\alpha=-\beta$.

Let us return to the general case. Since $L_{x}$ is of finite dimension, then there exists such number $N$ that $S_{0}, S_{1}, \ldots, S_{N}$ are linearly independent and

$$
S_{N+1}=\mu_{N} S_{N}+\mu_{N-1} S_{N-1}+\cdots+\mu_{0} S_{0} .
$$

Then

$$
D S_{N+1} D^{-1}=D\left(\mu_{N}\right) D S_{N} D^{-1}+D\left(\mu_{N-1}\right) D S_{N-1} D^{-1}+\cdots+D\left(\mu_{0}\right) D S_{0} D^{-1}
$$

and therefore,

$$
\begin{aligned}
& e^{(N \alpha+\beta) \tau}\left\{\left(\mu_{N} S_{N}+\mu_{N-1} S_{N-1}+\cdots\right)+A\left(c_{N+1} \alpha+d_{N+1} \beta\right) S_{N}+A\left(p_{N+1} A^{\prime}+q_{N+1} A\right) S_{N+1}+\cdots\right\} \\
& \quad=D\left(\mu_{N}\right)\left\{e^{((N-1) \alpha+\beta) \tau}\left(S_{N}+A\left(c_{N} \alpha+d_{N} \beta\right) S_{N-1}+\cdots\right)\right\}+D\left(\mu_{N-1}\right)\left\{e^{((N-2) \alpha+\beta) \tau} S_{N-1}+\cdots\right\}+\cdots .
\end{aligned}
$$

By comparing the coefficients before $S_{N}$ we have

$$
e^{(N \alpha+\beta) \tau}\left\{\mu_{N}+A\left(c_{N+1} \alpha+d_{N+1} \beta\right)\right\}=D\left(\mu_{N}\right) e^{((N-1) \alpha+\beta) \tau} .
$$

It follows that $\mu_{N}$ is a constant and then

$$
A\left(c_{N+1} \alpha+d_{N+1} \beta\right)=\mu_{N}\left(e^{-\alpha \tau}-1\right) .
$$

If $c_{N+1} \alpha+d_{N+1} \beta=N\{[(N-1) / 2] \alpha+\beta\} \neq 0$, then

$$
A(\tau)=C_{1}\left(e^{-\alpha \tau}-1\right)
$$

for some constant $C_{1}$.

If $c_{N+1} \alpha+d_{N+1} \beta=N\{[(N-1) / 2] \alpha+\beta\}=0$ (in this case $\mu_{N}=0$ ) we compare coefficients before $S_{N-1}$ and have

$$
e^{(N \alpha+\beta) \tau}\left\{\mu_{N-1}+A\left(p_{N+1} A^{\prime}+q_{N+1} A\right)\right\}=D\left(\mu_{N-1}\right) e^{((N-2) \alpha+\beta) \tau} .
$$

It follows that $\mu_{N-1}$ is a constant and

$$
p_{N+1} A A^{\prime}+q_{N+1} A^{2}=\mu_{N-1}\left(e^{-2 \alpha \tau}-1\right) .
$$

Note that if $c_{N+1} \alpha+d_{N+1} \beta=N\{[(N-1) / 2] \alpha+\beta\}=0$, then $p_{N+1}=-[N(N-1)(N+1) / 12] \alpha \neq 0$ and $q_{N+1}=-[(N-1) N(N+1) / 24] \alpha^{2} \neq 0$ for $N \geq 2$. Therefore, $\left(2 / q_{N+1}\right) p_{N+1}=\alpha$. Case $N=1$ should be studied separately $\left(S_{2}=\mu_{1} S_{1}+\mu_{0} S_{0}\right)$ and it was already. Let us solve the equation

$$
p_{N+1} A A^{\prime}+q_{N+1} A^{2}=\mu_{N-1}\left(e^{-2 \alpha \tau}-1\right) .
$$

Denote by $y=A^{2}$. We have 


$$
y^{\prime}+\alpha y=k_{1} e^{-2 \alpha \tau}-k_{1}
$$

for some constant $k_{1}$. It follows that

$$
A^{2}(\tau)=K_{1}\left(e^{-2 \alpha \tau}+K_{2} e^{-\alpha \tau}+1\right)
$$

for some constants $K_{1}$ and $K_{2}$.

Construct a new sequence of vector fields,

$$
S_{2}^{*}=\left[S_{1}, S_{0}\right], \quad S_{n+1}^{*}=\left[S_{1}, S_{n}^{*}\right], \quad n \geq 2 .
$$

Note that $S_{2}^{*}=-S_{2}$. Since $L_{x}$ is of finite dimension, then there exists number $M$ such that $S_{0}, S_{1}, \ldots, S_{M}^{*}$ are linearly independent and

$$
S_{M+1}^{*}=\mu_{M}^{*} S_{M}^{*}+\mu_{M-1}^{*} S_{M-1}^{*}+\cdots+\mu_{0}^{*} S_{0} .
$$

Following are the possibilities:

(2)

$$
\begin{aligned}
& A(\tau)=K_{1}\left(e^{-\alpha \tau}-1\right), \\
& B(\tau)=K_{3}\left(e^{-\beta \tau}-1\right),
\end{aligned}
$$

$$
\begin{gathered}
A(\tau)=K_{1}\left(e^{-\alpha \tau}-1\right), \\
B^{2}(\tau)=K_{3}^{2}\left(e^{-2 \beta \tau}+K_{4} e^{-\beta \tau}+1\right), \quad S_{M+1}^{*}=\mu_{M}^{*} S_{M}^{*}+\mu_{M-1}^{*} S_{M-1}^{*}+\cdots+\mu_{0}^{*} S_{0}, \quad \frac{M-1}{2} \beta+\alpha=0,
\end{gathered}
$$

(3)

$$
\begin{gathered}
B(\tau)=K_{3}\left(e^{-\beta \tau}-1\right), \\
A^{2}(\tau)=K_{1}^{2}\left(e^{-2 \alpha \tau}+K_{2} e^{-\alpha \tau}+1\right), \quad S_{N+1}=\mu_{N} S_{N}+\mu_{N-1} S_{N-1}+\cdots+\mu_{0} S_{0}, \quad \frac{N-1}{2} \alpha+\beta=0,
\end{gathered}
$$

and

(4)

$$
\begin{gathered}
A^{2}(\tau)=K_{1}^{2}\left(e^{-2 \alpha \tau}+K_{2} e^{-\alpha \tau}+1\right), \quad S_{N+1}=\mu_{N} S_{N}+\mu_{N-1} S_{N-1}+\cdots+\mu_{0} S_{0}, \quad \frac{N-1}{2} \alpha+\beta=0, \\
B^{2}(\tau)=K_{3}^{2}\left(e^{-2 \beta \tau}+K_{4} e^{-\beta \tau}+1\right), \quad S_{M+1}^{*}=\mu_{M}^{*} S_{M}^{*}+\mu_{M-1}^{*} S_{M-1}^{*}+\cdots+\mu_{0}^{*} S_{0}, \quad \frac{M-1}{2} \beta+\alpha=0,
\end{gathered}
$$

where $K_{1}, K_{2} \neq-2, K_{3}$, and $K_{4} \neq-2$ are some constants, $M, N \geq 2$.

In case (1), vector fields $S_{0}$ and $S_{1}$ generate an infinite dimensional Lie algebra $L_{x}$ unless $\alpha$ $+\beta=0$.

In case (2), we make a substitution $1-e^{\alpha \tau}=e^{-\alpha w}$. Vector fields $S_{0}$ and $S_{1}$ become

$$
S_{0}=K_{1} \frac{\partial}{\partial w}+\cdots
$$




$$
S_{1}=\left\{K_{3}^{2}\left(\left(1-e^{-\alpha w}\right)^{-2 \beta / \alpha}+K_{4}\left(1-e^{-\alpha w}\right)^{-\beta / \alpha}+1\right)\right\}^{1 / 2} \frac{\partial}{\partial w}+\cdots=g(w) \frac{\partial}{\partial w}+\cdots .
$$

Note that if

$$
S_{M+1}^{*}=\mu_{M}^{*} S_{M}^{*}+\mu_{M-1}^{*} S_{M-1}^{*}+\cdots+\mu_{0}^{*} S_{0},
$$

then all coefficients $\mu_{k}^{*}$ are constants. By comparing coefficients before $\partial / \partial w$ in both sides of the last equation we obtain that $g(w)$ is a solution of linear differential equation with constant coefficients, that is,

$$
g(w)=\left\{K_{3}^{2}\left(\left(1-e^{-\alpha w}\right)^{-2 \beta / \alpha}+K_{4}\left(1-e^{-\alpha w}\right)^{-\beta / \alpha}+1\right)\right\}^{1 / 2}=\sum_{k} R_{k}(w) e^{\nu_{k} w},
$$

where $R_{k}(w)$ are some polynomials. One can show that equality (45) holds only if $B(\tau)=K_{3}\left(e^{\alpha \tau}\right.$ $+1)$. It can be shown that in case $(3) A(\tau)=K_{1}\left(e^{\beta \tau}+1\right)$. In case (4) we make substitution $e^{\alpha \tau}$ $+K_{1} / 2+\sqrt{e^{2 \alpha \tau+K_{1} e^{\alpha \tau}+1}}=e^{\alpha w}$. Then

$$
\begin{gathered}
S_{0}=K_{1} \frac{\partial}{\partial w}+\cdots \\
\left.S_{1}=\left\{K_{3}^{2}\left(\frac{1}{2} e^{\alpha w}-\frac{K_{1}}{2}+\left(\frac{K_{1}^{2}}{8}-\frac{1}{2}\right) e^{-\alpha w}\right)^{-2 \beta / \alpha}+K_{4}\left(\frac{1}{2} e^{\alpha w}-\frac{K_{1}}{2}+\left(\frac{K_{1}^{2}}{8}-\frac{1}{2}\right) e^{-\alpha w}\right)^{-\beta / \alpha}+1\right)\right\}^{1 / 2} \frac{\partial}{\partial w} \\
+\cdots=g(w) \frac{\partial}{\partial w}+\cdots .
\end{gathered}
$$

For function $g(w)$ to be of form $\sum_{k} R_{k}(w) e^{\nu_{k} w}$, where $R_{k}(w)$ are polynomials, function $B(\tau)$ has to be of form $B(\tau)=K_{3}\left(e^{\alpha \tau}+1\right)$. Then, by case (3), $A(\tau)=K_{1}\left(e^{-\alpha \tau}+1\right)$.

It has been proved that in cases (1)-(4) one has

$\left(1^{*}\right)$

$$
\begin{aligned}
& A(\tau)=K_{1}\left(e^{-\alpha \tau}-1\right), \\
& B(\tau)=K_{3}\left(e^{\alpha \tau}-1\right),
\end{aligned}
$$

$$
\begin{aligned}
& A(\tau)=K_{1}\left(e^{-\alpha \tau}-1\right), \\
& B(\tau)=K_{3}\left(e^{\alpha \tau}+1\right),
\end{aligned}
$$

$$
\left\{\begin{array}{l}
A(\tau)=K_{1}\left(e^{-\alpha \tau}+1\right) \\
B(\tau)=K_{3}\left(e^{\alpha \tau}-1\right)
\end{array}\right.
$$

and

$$
\begin{aligned}
& A(\tau)=K_{1}\left(e^{-\alpha \tau}+1\right), \\
& B(\tau)=K_{3}\left(e^{\alpha \tau}+1\right) .
\end{aligned}
$$


In case $\left(1^{*}\right)$ function $d\left(t, t_{1}\right)$ in (12) has the form $d\left(t, t_{1}\right)=c_{4}\left(e^{\alpha t_{1}}-e^{\alpha t}\right)+c_{5}\left(e^{-\alpha t_{1}}-e^{-\alpha t}\right)$, where $c_{4}$ and $c_{5}$ are some constants. Equation (12) with such function $d\left(t, t_{1}\right)$ admits a nontrivial $x$-integral (see Sec. I, Theorem 3 and Sec. VIII).

In Secs. VI and VII we show that cases $\left(3^{*}\right)$ and $\left(4^{*}\right)$ both correspond to infinite dimensional Lie algebra $L_{x}$. Case $\left(2^{*}\right)$ also produces an infinite dimensional Lie algebra $L_{x}$. It can be proved in the same way as it is proved for case $\left(3^{*}\right)$.

\section{CHARACTERISTIC LIE ALGEBRA $L_{x}$ OF THE CHAIN $t_{1 x}=t_{x}+A_{1}\left(e^{\alpha t_{1}}+e^{\alpha t}\right)$ $-A_{2}\left(e^{-\alpha t}-e^{-\alpha t_{1}}\right)$}

Since $A(\tau)=A_{1}\left(e^{-\alpha \tau}+1\right)$ and $B(\tau)=A_{2}\left(e^{\alpha \tau}-1\right)$, then

$$
A(\tau) e^{\alpha t}+\sum_{j=1}^{k} A\left(\tau_{j}\right) e^{\alpha t_{j}}=A_{1}\left(e^{\alpha t}+\left(2 \sum_{j=1}^{k-1} e^{\alpha t_{j}}\right)+e^{\alpha t_{k}}\right)
$$

and

$$
B(\tau) e^{-\alpha t}+\sum_{j=1}^{k} B\left(\tau_{j}\right) e^{-\alpha t_{j}}=A_{2}\left(e^{-\alpha t}-e^{-\alpha t_{k}}\right)
$$

We have

$$
\frac{1}{A_{1}} S_{0}=\left(e^{\alpha t}+e^{\alpha t_{1}}\right) \frac{\partial}{\partial t_{1}}+\sum_{k=1}^{\infty}\left(e^{\alpha t}+\left(2 \sum_{j=1}^{k-1} e^{\alpha t_{j}}\right)+e^{\alpha t_{k}}\right) \frac{\partial}{\partial t_{k}}+\sum_{k=1}^{\infty}\left(e^{\alpha t}+\left(2 \sum_{j=1}^{k-1} e^{\alpha t_{-j}}\right)+e^{\alpha t_{-k}}\right) \frac{\partial}{\partial t_{-k}}
$$

and

$$
\frac{1}{A_{2}} S_{1}=e^{-\alpha \tau} \tilde{X}-\sum_{k=-\infty}^{\infty} e^{-\alpha t_{k}} \frac{\partial}{\partial t_{k}}=e^{-\alpha \tau} \tilde{X}-\tilde{S}_{1}
$$

where

$$
\tilde{S}_{1}=\sum_{k=-\infty}^{\infty} e^{-\alpha t_{k}} \frac{\partial}{\partial t_{k}}
$$

In variables $w_{j}=(1 / \alpha) e^{\alpha t_{j}}$ vector fields $\widetilde{S}_{1}$ and $\left(1 / A_{1}\right) S_{0}$ can be rewritten as

$$
\begin{gathered}
\tilde{S}_{1}=\sum_{k=-\infty}^{\infty} \frac{\partial}{\partial w_{j}}, \\
\frac{1}{A_{1}} S_{0}=\alpha^{2} \sum_{k=1}^{\infty}\left\{w_{k}\left(w+2 \sum_{j=1}^{k-1} w_{j}\right)+w_{k}^{2}\right\} \frac{\partial}{\partial w_{k}}+\alpha^{2} \sum_{k=1}^{\infty}\left\{w_{-k}\left(w+2 \sum_{j=1}^{k-1} w_{-j}\right)+w_{-k}^{2}\right\} \frac{\partial}{\partial w_{-k}} .
\end{gathered}
$$

We have

$$
\begin{gathered}
T_{1}=\left[\tilde{S}_{1},\left[\tilde{S}_{1}, \frac{1}{\alpha^{2} A_{1}} S_{0}\right]\right]=4 \sum_{k=-\infty}^{\infty} k \frac{\partial}{\partial w_{k}}=4 \widetilde{T}_{1}, \quad \widetilde{T}_{1}=\sum_{k=-\infty}^{\infty} k \frac{\partial}{\partial w_{k}}, \\
T_{2}=\left[\tilde{S}_{1},\left[\tilde{T}_{1}, \frac{1}{\alpha^{2} A_{1}} S_{0}\right]\right]=3 \sum_{k=1}^{\infty}\left\{k^{2}-k+1\right\}\left(\frac{\partial}{\partial w_{k}}+\frac{\partial}{\partial w_{-k}}\right)=3 \widetilde{T}_{2}-3 \widetilde{T}_{1}+3 \tilde{S}_{1}, \quad \widetilde{T}_{2}=\sum_{k=-\infty}^{\infty} k^{2} \frac{\partial}{\partial w_{k}} .
\end{gathered}
$$

Assume that $\widetilde{T}_{m}=\sum_{k=-\infty}^{\infty} k^{m}\left(\partial / \partial w_{k}\right), m=1,2 \ldots, n$, are vector fields from $L_{x}$. Then 


$$
\begin{aligned}
T_{m+1} & =\left[\tilde{S}_{1},\left[\widetilde{T}_{m}, \frac{1}{\alpha^{2} A_{1}} S_{0}\right]\right]=\sum_{k=1}^{\infty}\left\{2\left(1+2^{m}+3^{m}+\cdots+k^{m}\right)+2 k^{m+1}-k^{m}\right\}\left(\frac{\partial}{\partial w_{k}}+\frac{\partial}{\partial w_{-k}}\right) \\
& =\sum_{k=1}^{\infty}\left\{2\left(\frac{k^{m+1}}{m+1}+d_{m, m+1} k^{m}+\cdots+d_{1, m+1} k+d_{0, m+1}\right)+2 k^{m+1}-k^{m}\right\}\left(\frac{\partial}{\partial w_{k}}+\frac{\partial}{\partial w_{-k}}\right)
\end{aligned}
$$

and therefore, $\widetilde{T}_{m+1}=\sum_{k=-\infty}^{\infty} k^{m+1} \frac{\partial}{\partial w_{k}} \in L_{x}$. It shows that $\widetilde{T}_{n}=\sum_{k=-\infty}^{\infty} k^{n} \frac{\partial}{\partial w_{k}} \in L_{x}$ for all $n=1,2,3, \ldots$ and $L_{x}$ is of infinite dimension.

\section{CHARACTERISTIC LIE ALGEBRA $L_{x}$ OF THE CHAIN $t_{1 x}=t_{x}+A_{1}\left(e^{\alpha t_{1}}+e^{\alpha t}\right)$ $+A_{2}\left(e^{-\alpha t}+e^{-\alpha t_{1}}\right)$}

It was observed in previous studies (see, for instance, Ref. 10) that $S$-integrable models have the characteristic Lie algebra of finite growth. The chain studied in this section can easily be reduced to the semidiscrete SG model $t_{1 x}=t_{x}+\sin t+\sin t_{1}$ which belongs to the $S$-integrable class. It is remarkable that its algebra $L_{x}$ is of finite growth, or, more exactly, the dimension of the linear space of multiple commutators grows linearly with the multiplicity. Below we prove that the linear space $V_{n}$ of all commutators of multiplicity $\leq n$ has a basis $\left\{P_{1}, P_{2}, P_{3}, \ldots P_{2 k} ; Q_{2}, Q_{4}, \ldots Q_{2 k}\right\}$ for $n=2 k$ and a basis $\left\{P_{1}, P_{2}, P_{3}, \ldots P_{2 k+1} ; Q_{2}, Q_{4}, \ldots Q_{2 k}\right\}$ for $n=2 k+1$, where the operators $P_{j}$ and $Q_{j}$ are defined consecutively,

$$
\begin{gathered}
P_{1}=\left[S_{0}, S_{1}\right]+\alpha S_{0}+\alpha S_{1}, \quad Q_{1}=P_{1}, \\
P_{2}=\left[S_{1}, P_{1}\right], \quad Q_{2}=\left[S_{0}, Q_{1}\right], \\
P_{3}=\left[S_{0}, P_{2}\right]+\alpha P_{2}, \quad Q_{3}=\left[S_{1}, Q_{2}\right]-\alpha Q_{2}, \\
P_{2 n}=\left[S_{1}, P_{2 n-1}\right], \quad Q_{2 n}=\left[S_{0}, Q_{2 n-1}\right], \\
P_{2 n+1}=\left[S_{0}, P_{2 n}\right]+\alpha P_{2 n}, \quad Q_{2 n+1}=\left[S_{1}, Q_{2 n}\right]-\alpha Q_{2 n}
\end{gathered}
$$

for $n \geq 1$. Direct calculations show that

$$
\begin{gathered}
D P_{1} D^{-1}=P_{1}-2 \alpha\left(S_{0}+S_{1}\right), \\
D P_{2} D^{-1}=e^{-\alpha \tau}\left(P_{2}+2 \alpha P_{1}-2 \alpha^{2}\left(S_{0}+S_{1}\right)\right), \\
D P_{3} D^{-1}=P_{3}+2 \alpha Q_{2}-2 \alpha P_{2}-4 \alpha^{2} P_{1}+4 \alpha^{3}\left(S_{0}+S_{1}\right), \\
D P_{4} D^{-1}=e^{-\alpha \tau}\left(P_{4}+2 \alpha Q_{3}-4 \alpha^{2} P_{2}+4 \alpha^{2} Q_{2}-4 \alpha^{3} P_{1}+4 \alpha^{4}\left(S_{0}+S_{1}\right)\right), \\
D Q_{2} D^{-1}=e^{\alpha \tau}\left(Q_{2}-2 \alpha P_{1}+2 \alpha^{2}\left(S_{0}+S_{1}\right)\right), \\
D Q_{3} D^{-1}=Q_{3}+2 \alpha Q_{2}-2 \alpha P_{2}-4 \alpha^{2} P_{1}+4 \alpha^{3}\left(S_{0}+S_{1}\right), \\
P_{3}=Q_{3}, \quad\left[S_{1}, P_{2}\right]=-\alpha P_{2},\left[S_{0}, Q_{2}\right]=\alpha Q_{2},\left[S_{1}, P_{4}\right]=-\alpha P_{4},\left[S_{0}, Q_{4}\right]=\alpha Q_{4} .
\end{gathered}
$$

The coefficient before $\partial / \partial \tau$ in all vector fields $D P_{i} D^{-1}$ and $D Q_{i} D^{-1}, 1 \leq i \leq 4$, is zero. 
Lemma 10: For $n \geq 1$ we have

(1) $D P_{2 n+1} D^{-1}+2 \alpha e^{\alpha \tau} D P_{2 n} D^{-1}=P_{2 n+1}+2 \alpha Q_{2 n}$,

(2) $e^{\alpha \tau} D P_{2 n+2} D^{-1}-\alpha D P_{2 n+1} D^{-1}=P_{2 n+2}+\alpha Q_{2 n+1}$,

(3) $D Q_{2 n+1} D^{-1}-2 \alpha e^{-\alpha \tau} D Q_{2 n} D^{-1}=Q_{2 n+1}-2 \alpha P_{2 n}$,

(4) $e^{-\alpha \tau} D Q_{2 n+2} D^{-1}+\alpha D Q_{2 n+1} D^{-1}=Q_{2 n+2}-\alpha P_{2 n+1}$,

(5) $P_{2 n+1}=Q_{2 n+1}$,

(6) $\left[S_{1}, P_{2 n+2}\right]=-\alpha P_{2 n+2}$, and

(7) $\left[S_{0}, Q_{2 n+2}\right]=\alpha Q_{2 n+2}$.

Moreover, the coefficient before $\partial / \partial \tau$ in all vector fields $D P_{k} D^{-1}$ and $D Q_{k} D^{-1}$ is zero.

Proof: We prove the lemma by induction on $n$. It follows from (46) that the base of induction holds for $n=1$. Assume that (1)-(7) are true for all $n, 1 \leq n \leq k$. Let us prove that (1) is true for $n=k+1$.

$$
\begin{aligned}
D P_{2 n+3} D^{-1}= & D\left(\left[S_{0}, P_{2 n+2}\right]+\alpha P_{2 n+2}\right) D^{-1}=\left[e^{\alpha \tau} S_{0}, D P_{2 n+2} D^{-1}\right]+\alpha D P_{2 n+2} D^{-1} \\
= & {\left[e^{\alpha \tau} S_{0}, \alpha e^{-\alpha \tau} D P_{2 n+1} D^{-1}+e^{-\alpha \tau} P_{2 n+2}+\alpha e^{-\alpha \tau} Q_{2 n+1}\right]+\alpha D P_{2 n+2} D^{-1} } \\
= & -\alpha^{2}\left(1+e^{-\alpha \tau}\right) D P_{2 n+1} D^{-1}+\alpha e^{-\alpha \tau}\left[e^{\alpha \tau} S_{0}, D P_{2 n+1} D^{-1}\right] \\
& -\alpha\left(1+e^{-\alpha \tau}\right) P_{2 n+2}-\alpha^{2}\left(1+e^{-\alpha \tau}\right) Q_{2 n+1}+P_{2 n+3}-\alpha P_{2 n+2}+\alpha Q_{2 n+2}+\alpha D P_{2 n+2} D^{-1} \\
= & -\alpha^{2}\left(1+e^{-\alpha \tau}\right) D P_{2 n+1} D^{-1}+\alpha e^{-\alpha \tau} D\left[S_{0}, Q_{2 n+1}\right] D^{-1}-\alpha\left(2+e^{-\alpha \tau}\right) P_{2 n+2} \\
& -\alpha^{2}\left(1+e^{-\alpha \tau}\right) Q_{2 n+1}+P_{2 n+3}+\alpha Q_{2 n+2}+\alpha D P_{2 n+2} D^{-1} \\
= & -\alpha^{2}\left(1+e^{-\alpha \tau}\right) D P_{2 n+1} D^{-1}+\alpha Q_{2 n+2}-\alpha^{2} P_{2 n+1}-\alpha^{2} D Q_{2 n+1} D^{-1} \\
& -\alpha\left(2+e^{-\alpha \tau}\right) P_{2 n+2}-\alpha^{2}\left(1+e^{-\alpha \tau}\right) Q_{2 n+1}-2 \alpha^{2} Q_{2 n+1}-2 \alpha P_{2 n+2}+P_{2 n+3} \\
= & -2 \alpha^{2} D P_{2 n+1} D^{-1}+2 \alpha Q_{2 n+2}-2 \alpha^{2} Q_{2 n+1}-2 \alpha P_{2 n+2}+P_{2 n+3} \\
= & 2 \alpha P_{2 n+2}+2 \alpha^{2} Q_{2 n+1}-2 \alpha e^{\alpha \tau} D P_{2 n+2} D^{-1}+2 \alpha Q_{2 n+2}-2 \alpha^{2} Q_{2 n+1}-2 \alpha P_{2 n+2}+P_{2 n+3} \\
= & -2 \alpha e^{\alpha \tau} D P_{2 n+2} D^{-1}+2 \alpha Q_{2 n+2}+P_{2 n+3} .
\end{aligned}
$$

The proof of (3) is the same as the proof of (1). Let us show that (5) is true for $n=k+1$. We have

$$
\begin{aligned}
D P_{2 n+3} D^{-1}= & -2 \alpha e^{\alpha \tau} D P_{2 n+2} D^{-1}+2 \alpha Q_{2 n+2}+P_{2 n+3}=-2 \alpha\left(\alpha D P_{2 n+1} D^{-1}+P_{2 n+2}+\alpha Q_{2 n+1}\right) \\
& +2 \alpha Q_{2 n+2}+P_{2 n+3}
\end{aligned}
$$

and

$$
\begin{aligned}
D Q_{2 n+3} D^{-1}= & 2 \alpha e^{-\alpha \tau} D Q_{2 n+2} D^{-1}-2 \alpha P_{2 n+2}+Q_{2 n+3}=2 \alpha\left(-\alpha D Q_{2 n+1} D^{-1}+Q_{2 n+2}-\alpha P_{2 n+1}\right) \\
& -2 \alpha P_{2 n+2}+Q_{2 n+3} .
\end{aligned}
$$

By (5), $P_{2 n+1}=Q_{2 n+1}$ and therefore

$$
D\left(P_{2 n+3}-Q_{2 n+3}\right) D^{-1}=-2 \alpha P_{2 n+2}-2 \alpha Q_{2 n+2}+2 \alpha Q_{2 n+2}+2 \alpha P_{2 n+2}=0 .
$$

Hence, $P_{2 n+3}=Q_{2 n+3}$. 
Let us prove that (2) is true for $n=k+1$. We have

$$
\begin{aligned}
e^{\alpha \tau} D P_{2 n+1} D^{-1}= & e^{\alpha \tau} D\left[S_{1}, P_{2 n+3}\right] D^{-1}=e^{\alpha \tau}\left[e^{-\alpha \tau} S_{1}, D P_{2 n+3} D^{-1}\right] \\
= & e^{\alpha \tau}\left[e^{-\alpha \tau} S_{1},-2 \alpha e^{\alpha \tau} D P_{2 n+2} D^{-1}+2 \alpha Q_{2 n+2}+P_{2 n+3}\right] \\
= & e^{\alpha \tau}\left(-2 \alpha^{2}\left(1+e^{\alpha \tau}\right) D P_{2 n+2} D^{-1}\right)-2 \alpha e^{2 \alpha \tau}\left[e^{-\alpha \tau} S_{1}, D P_{2 n+2} D^{-1}\right]+P_{2 n+4}+2 \alpha Q_{2 n+3} \\
& +2 \alpha^{2} Q_{2 n+2} \\
= & -2 \alpha^{2}\left(e^{\alpha \tau}+e^{2 \alpha \tau}\right) D P_{2 n+2} D^{-1}+2 \alpha^{2} e^{2 \alpha \tau} D P_{2 n+2} D^{-1}+P_{2 n+4}+2 \alpha Q_{2 n+3}+2 \alpha^{2} Q_{2 n+2} \\
= & -2 \alpha^{2} e^{\alpha \tau} D P_{2 n+2} D^{-1}+P_{2 n+4}+2 \alpha Q_{2 n+3}+2 \alpha^{2} Q_{2 n+2} \\
= & \alpha D P_{2 n+3} D^{-1}-\alpha P_{2 n+3}-2 \alpha^{2} Q_{2 n+2}+P_{2 n+4}+2 \alpha Q_{2 n+3}+2 \alpha^{2} Q_{2 n+2} \\
= & \alpha D P_{2 n+3} D^{-1}+\alpha Q_{2 n+3}+P_{2 n+4} .
\end{aligned}
$$

The proof of (4) is similar to the proof of (2).

Let us prove that (6) is true for $n=k+1$,

$$
\begin{aligned}
D\left[S_{1}, P_{2 n+4}\right] D^{-1}= & {\left[e^{-\alpha \tau} S_{1}, \alpha e^{-\alpha \tau} D P_{2 n+3} D^{-1}+e^{-\alpha \tau} P_{2 n+4}+\alpha e^{-\alpha \tau} Q_{2 n+3}\right] } \\
= & {\left[e^{-\alpha \tau} S_{1}, \alpha e^{-\alpha \tau}\left(-2 \alpha e^{\alpha \tau} D P_{2 n+2} D^{-1}+P_{2 n+3}+2 \alpha Q_{2 n+2}\right)+e^{-\alpha \tau} P_{2 n+4}+\alpha \mathrm{e}^{-\alpha \tau} Q_{2 n+3}\right] } \\
= & {\left[e^{-\alpha \tau} S_{1},-2 \alpha^{2} D P_{2 n+2} D^{-1}+2 \alpha e^{-\alpha \tau} P_{2 n+3}+2 \alpha^{2} e^{-\alpha \tau} Q_{2 n+2}+e^{-\alpha \tau} P_{2 n+4}\right] } \\
= & -2 \alpha^{2} D\left[S_{1}, P_{2 n+2}\right] D^{-1}-2 \alpha^{2} e^{-2 \alpha \tau}\left(1+e^{\alpha \tau}\right) P_{2 n+3}-2 \alpha^{3} e^{-2 \alpha \tau}\left(1+e^{\alpha \tau}\right) Q_{2 n+2} \\
& +2 \alpha e^{-2 \alpha \tau} P_{2 n+4}+2 \alpha^{2} e^{-2 \alpha \tau} Q_{2 n+3}+2 \alpha^{3} e^{-2 \alpha \tau} Q_{2 n+2}-\alpha e^{-2 \alpha \tau}\left(1+e^{\alpha \tau}\right) P_{2 n+4} \\
& +\mathrm{e}^{-2 \alpha \tau}\left[S_{1}, P_{2 n+4}\right] \\
= & 2 \alpha^{3} D P_{2 n+2} D^{-1}-2 \alpha^{2} e^{-\alpha \tau} P_{2 n+3}+\alpha\left(e^{-2 \alpha \tau}-e^{-\alpha \tau}\right) P_{2 n+4}-2 \alpha^{3} e^{-\alpha \tau} Q_{2 n+2} \\
& +\mathrm{e}^{-2 \alpha \tau}\left[S_{1}, P_{2 n+4}\right] \\
= & \alpha^{2} e^{-\alpha \tau} P_{2 n+3}+2 \alpha^{3} e^{-\alpha \tau} Q_{2 n+2}-\alpha^{2} e^{-\alpha \tau} D P_{2 n+3} D^{-1}-2 \alpha^{2} e^{-\alpha \tau} P_{2 n+3} \\
& +\alpha\left(e^{-2 \alpha \tau}-e^{-\alpha \tau}\right) P_{2 n+4}-2 \alpha^{3} e^{-\alpha \tau} Q_{2 n+2}+e^{-2 \alpha \tau}\left[S_{1}, P_{2 n+4}\right] \\
= & -\alpha^{2} e^{-\alpha \tau} P_{2 n+3}+\alpha\left(e^{-2 \alpha \tau}-e^{-\alpha \tau}\right) P_{2 n+4}-\alpha D P_{2 n+4} D^{-1}+\alpha e^{-\alpha \tau} P_{2 n+4}+\alpha^{2} e^{-\alpha \tau} Q_{2 n+3} \\
& +e^{-2 \alpha \tau}\left[S_{1}, P_{2 n+4}\right] .
\end{aligned}
$$

Thus,

$$
\begin{gathered}
D\left[S_{1}, P_{2 n+4}\right] D^{-1}=e^{-2 \alpha \tau}\left[S_{1}, P_{2 n+4}\right]+\alpha e^{-2 \alpha \tau} P_{2 n+4}-\alpha D P_{2 n+4} D^{-1}, \\
D\left(\left[S_{1}, P_{2 n+4}\right]+\alpha P_{2 n+4}\right) D^{-1}=e^{-2 \alpha \tau}\left(\left[S_{1}, P_{2 n+4}\right]+\alpha P_{2 n+4}\right) .
\end{gathered}
$$

Hence, $\left[S_{1}, P_{2 n+4}\right]=-\alpha P_{2 n+4}$.

The proof of (7) is similar to the proof of (6).

Corollary 1: We have

$$
\begin{gathered}
e^{-\alpha \tau} D Q_{2 n} D^{-1}+e^{\alpha \tau} D P_{2 n} D^{-1}=Q_{2 n}+P_{2 n}, \\
D P_{2 n+1} D^{-1}=P_{2 n+1}+\sum_{k=1}^{n}\left(\mu_{2 k}^{(2 n+1)} P_{2 k}+\nu_{2 k}^{(2 n+1)} Q_{2 k}\right)+\sum_{k=0}^{n-1} \mu_{2 k+1}^{(2 n+1)} P_{2 k+1}+\mu_{0}^{(2 n+1)} S_{0}+\nu_{0}^{(2 n+1)} S_{1}, \\
D P_{2 n} D^{-1}=e^{-\alpha \tau}\left(P_{2 n}+\sum_{k=1}^{n-1}\left(\mu_{2 k}^{(2 n)} P_{2 k}+\nu_{2 k}^{(2 n)} Q_{2 k}\right)+\sum_{k=0}^{n-1} \mu_{2 k+1}^{(2 n)} P_{2 k+1}+\mu_{0}^{(2 n)} S_{0}+\nu_{0}^{(2 n)} S_{1}\right),
\end{gathered}
$$




$$
D Q_{2 n} D^{-1}=e^{\alpha \tau}\left(Q_{2 n}-\sum_{k=1}^{n-1}\left(\mu_{2 k}^{(2 n)} P_{2 k}+\nu_{2 k}^{(2 n)} Q_{2 k}\right)-\sum_{k=0}^{n-1} \mu_{2 k+1}^{(2 n)} P_{2 k+1}-\mu_{0}^{(2 n)} S_{0}-\nu_{0}^{(2 n)} S_{1}\right) .
$$

Moreover, $\mu_{2 n}^{(2 n+1)}=-2 \alpha, \nu_{2 n}^{(2 n+1)}=2 \alpha$, and $\mu_{2 n-1}^{(2 n)}=2 \alpha$.

Assume that $L_{x}$ is of finite dimension. There are three possibilities:

(1) $S_{0}, S_{1}, P_{1}, P_{2}, Q_{2}, P_{3}, P_{4}, Q_{4}, \ldots, P_{2 n-1}$ are linearly independent and

$S_{0}, S_{1}, P_{1}, P_{2}, Q_{2}, P_{3}, P_{4}, Q_{4}, \ldots, P_{2 n-1}, P_{2 n}$ are linearly dependent,

(2) $S_{0}, S_{1}, P_{1}, P_{2}, Q_{2}, P_{3}, P_{4}, Q_{4}, \ldots, P_{2 n-1}, P_{2 n}$ are linearly independent and

$S_{0}, S_{1}, P_{1}, P_{2}, Q_{2}, P_{3}, P_{4}, Q_{4}, \ldots, P_{2 n-1}, P_{2 n}, Q_{2 n}$ are linearly dependent, and

(3) $S_{0}, S_{1}, P_{1}, P_{2}, Q_{2}, P_{3}, P_{4}, Q_{4}, \ldots, P_{2 n}, Q_{2 n}$ are linearly independent and

$S_{0}, S_{1}, P_{1}, P_{2}, Q_{2}, P_{3}, P_{4}, Q_{4}, \ldots, P_{2 n}, Q_{2 n}, P_{2 n+1}$ are linearly dependent.

In case (1),

$$
P_{2 n}=\gamma_{2 n-1} P_{2 n-1}+\gamma_{2 n-2} P_{2 n-2}+\eta_{2 n-2} Q_{2 n-2}+\cdots
$$

and

$$
D P_{2 n} D^{-1}=D\left(\gamma_{2 n-1}\right) D P_{2 n-1} D^{-1}+D\left(\gamma_{2 n-2}\right) D P_{2 n-2} D^{-1}+D\left(\eta_{2 n-2}\right) D Q_{2 n-2} D^{-1}+\cdots
$$

We use Corollary 1 to compare the coefficients before $P_{2 n-1}$ in (47) and have the contradictory equality

$$
e^{-\alpha \tau}\left(\gamma_{2 n-1}+2 \alpha\right)=D\left(\gamma_{2 n-1}\right)
$$

It shows that case (1) is impossible to have.

In case (2),

$$
Q_{2 n}=\gamma_{2 n} P_{2 n}+\gamma_{2 n-1} P_{2 n-1}+\eta_{2 n-2} Q_{2 n-2}+\cdots
$$

and

$$
D Q_{2 n} D^{-1}=D\left(\gamma_{2 n}\right) D P_{2 n} D^{-1}+D\left(\gamma_{2 n-1}\right) D P_{2 n-1} D^{-1}+D\left(\eta_{2 n-2}\right) D Q_{2 n-2} D^{-1}+\cdots
$$

We use Corollary 1 to compare the coefficients before $P_{2 n-1}$ in (48) and have the contradictory equation

$$
e^{\alpha \tau}\left(\gamma_{2 n-1}-2 \alpha\right)=D\left(\gamma_{2 n-1}\right)
$$

It shows that case (2) is impossible to have.

In case (3),

$$
P_{2 n+1}=\eta_{2 n} Q_{2 n}+\gamma_{2 n} P_{2 n}+\cdots
$$

and

$$
D P_{2 n+1} D^{-1}=D\left(\eta_{2 n}\right) D Q_{2 n} D^{-1}+D\left(\gamma_{2 n}\right) D P_{2 n} D^{-1}+\cdots
$$

We use Corollary 1 to compare the coefficients before $P_{2 n}$ in (49) and have the contradictory equation

$$
\left(\gamma_{2 n}-2 \alpha\right)=D\left(\gamma_{2 n}\right) e^{-\alpha \tau}
$$

It shows that case (3) also fails to be true. Therefore, characteristic Lie algebra $L_{x}$ is of infinite dimension. 


\section{FINDING $x$-INTEGRALS}

Now we are ready to prove the main theorem, Theorem 3, formulated in Sec. I. Really, in the previous sections we proved that if chain (12) admits a nontrivial $x$-integral, then it is one of forms (1)-(4). List (i)-(iv) allows one to prove the inverse statement: each of the equations from the list admits indeed a nontrivial $x$-integral.

Let us explain briefly how we found list (i)-(iv). Since for each equation (1)-(4) we have constructed the related characteristic Lie algebra to find $x$-integral $F$ one has to solve the corresponding system of the first order partial differential equations. Below we illustrate the method with case (2), for which the basis of the characteristic algebra $L_{x}$ is given by the vector fields

$$
\tilde{Y}=\partial_{x}+Y_{a(\tau) t+b(\tau)}, \quad T_{1}=Y_{-a(\tau)}, \quad \tilde{X}=\frac{\partial}{\partial t}+\frac{\partial}{\partial t_{1}}+\frac{\partial}{\partial t_{-1}}+\frac{\partial}{\partial t_{2}}+\frac{\partial}{\partial t_{-2}}+\cdots,
$$

where $a(\tau)=c_{0} \tau$ and $b(\tau)=c_{2} \tau^{2}+c_{3} \tau$. Note that $x$-integral $F$ of (2) should satisfy the equations $\widetilde{Y} F=0, T_{1} F=0$, and $\tilde{X} F=0$. Introduce new variables $t, w, w_{ \pm 1}, \ldots$ where $w_{j}=\ln \left(\tau_{j}\right)$ and $\tau_{j}=t_{j}$ $-t_{j+1}$. Vector fields $\widetilde{X}, T_{1}$, and $\widetilde{Y}$ in new variables are rewritten as

$$
\begin{gathered}
\tilde{X}=\frac{\partial}{\partial t}, \quad T_{1}=\sum_{j=-\infty}^{\infty} c_{0} \frac{\partial}{\partial w_{j}}, \\
\tilde{Y}=\frac{\partial}{\partial x}-t \sum_{j=-\infty}^{\infty} c_{0} \frac{\partial}{\partial w_{j}}+c_{0} \sum_{j=-\infty}^{\infty}\left\{\widetilde{\rho}_{j}+\widetilde{b}\left(w_{j}\right)\right\} \frac{\partial}{\partial w_{j}}=\frac{\partial}{\partial x}-t T_{1}+c_{0} \sum_{j=-\infty}^{\infty}\left\{\widetilde{\rho}_{j}+\widetilde{b}\left(w_{j}\right)\right\} \frac{\partial}{\partial w_{j}},
\end{gathered}
$$

where

$$
\tilde{\rho}_{j}= \begin{cases}\sum_{k=0}^{j-1} e^{w_{k}} & \text { if } j \geq 1 \\ 0 & \text { if } j=0, \quad \tilde{b}\left(w_{j}\right)=-\frac{1}{c_{0}}\left(c_{2} e^{w_{j}}+c_{3}\right), \\ -\sum_{k=j}^{1} e^{w_{k}} & \text { if } j \leq-1 .\end{cases}
$$

Note that since we have $\tilde{X} F=0, F$ does not depend on $t$. Now let us consider the vector field

$$
\tilde{Y}+t T_{1}=A=\frac{\partial}{\partial x}+c_{0} \sum_{j=-\infty}^{\infty}\left\{\widetilde{\rho}_{j}+\tilde{b}\left(w_{j}\right)\right\} \frac{\partial}{\partial w_{j}} .
$$

We can write the vector field $A$ explicitly as

$$
A=\frac{\partial}{\partial x}+\sum_{j=-\infty}^{\infty}\left\{\left(c_{0} \sum_{k=0}^{j-1} e^{w_{k}}\right)-c_{2} e^{w_{j}}-c_{3}\right\} \frac{\partial}{\partial w_{j}}=\frac{\partial}{\partial x}-\frac{c_{3}}{c_{0}} T_{1}+\sum_{j=-\infty}^{\infty}\left\{\left(c_{0} \sum_{k=0}^{j-1} e^{w_{k}}\right)-c_{2} e^{w_{j}}\right\} \frac{\partial}{\partial w_{j}} .
$$

The commutator $\left[T_{1}, A\right]$ gives

$$
\left[T_{1}, A\right]=c_{0} A-c_{0} \frac{\partial}{\partial x}+c_{3} T_{1} .
$$

Thus we have three vector fields,

$$
A-\frac{\partial}{\partial x}+\frac{c_{3}}{c_{0}} T_{1}:=\tilde{A}=\sum_{j=-\infty}^{\infty}\left\{\left(c_{0} \sum_{k=0}^{j-1} e^{w_{k}}\right)-c_{2} e^{w_{j}}\right\} \frac{\partial}{\partial w_{j}},
$$




$$
\frac{T_{1}}{c_{0}}:=T_{1}=\sum_{j=-\infty}^{\infty} \frac{\partial}{\partial w_{j}}, \quad \tilde{X}_{1}=\frac{\partial}{\partial x},
$$

which solve $\widetilde{A} F=0, \widetilde{T}_{1} F=0$, and $\widetilde{X}_{1} F=0$. Note that $\left[\widetilde{T}_{1}, \widetilde{A}\right]=\widetilde{A}$. Since $\widetilde{X}_{1} F=0, F$ does not depend on $x$. Hence we end up with two equations. By the Jacobi theorem the system of equations has a nontrivial solution $F\left(w, w_{1}, w_{2}\right)$ depending on three variables. Therefore we need first three terms of $\tilde{A}$ and $\widetilde{T}_{1}$,

$$
\begin{gathered}
\tilde{A}=-c_{2} w \frac{\partial}{\partial w}+\left(c_{0} e^{w}-c_{2} e^{w_{1}}\right) \frac{\partial}{\partial w_{1}}+\left(c_{0} e^{w}+c_{0} e^{w_{1}}-c_{2} e^{w_{2}}\right) \frac{\partial}{\partial w_{2}}, \\
\widetilde{T}_{1}=\frac{\partial}{\partial w}+\frac{\partial}{\partial w_{1}}+\frac{\partial}{\partial w_{2}} .
\end{gathered}
$$

Now we again introduce new variables $w=\epsilon, w-w_{1}=\epsilon_{1}$, and $w_{1}-w_{2}=\epsilon_{2}$. Vector fields $\widetilde{A}$ and $\widetilde{T}_{1}$ in new variables are rewritten as

$$
\tilde{A}=e^{\epsilon}\left\{-c_{2} \frac{\partial}{\partial \epsilon}+\left(\left(-c_{2}-c_{0}\right)+c_{2} e^{-\epsilon_{1}}\right) \frac{\partial}{\partial \epsilon_{1}}+\left(\left(-c_{2}-c_{0}\right) e^{-\epsilon_{1}}+c_{2} e^{-\epsilon_{1}-\epsilon_{2}}\right) \frac{\partial}{\partial \epsilon_{2}}\right\}, \quad \widetilde{T}_{1}=\frac{\partial}{\partial \epsilon} .
$$

To find the $x$-integral (ii) in Theorem 3 one has to solve the equation

$$
\left\{\left(\left(-c_{2}-c_{0}\right)+c_{2} e^{-\epsilon_{1}}\right) \frac{\partial}{\partial \epsilon_{1}}+e^{-\epsilon_{1}}\left(\left(-c_{2}-c_{0}\right)+c_{2} e^{-\epsilon_{2}}\right) \frac{\partial}{\partial \epsilon_{2}}\right\} F=0 .
$$

\section{CONCLUSION}

In this article the problem of classification of Darboux integrable nonlinear semidiscrete chains of hyperbolic type was studied. An approach based on the notion of characteristic Lie algebra was properly modified and successfully used. We gave a complete list of hyperbolic-type chains $t_{1 x}=t_{x}+d\left(t, t_{1}\right)$ admitting nontrivial $x$-integrals. We demonstrated that the method of characteristic Lie algebras provides an effective tool to classify integrable discrete chains as well. The method did not get much attention in the literature. To our knowledge there are only two studies (see Refs. 9 and 14) where the characteristic Lie algebras are applied for solving the classification problem for the partial differential equations and systems. Surprisingly the first of them was published in 1981 and the second one only 25 years later.

\section{ACKNOWLEDGMENTS}

This work is partially supported by the Scientific and Technological Research Council of Turkey (TÜBITAK). One of the authors (I.H.) thanks also the Russian Foundation for Basic Research (RFBR) (Grant Nos. 06-01-92051KE-a and 08-01-00440-a).

${ }^{1}$ V. E. Adler and S. Y. Startsev, Teor. Mat. Fiz. 121, 271 (1999); [ Theor. Math. Phys. 121, 1484 (1999)]

${ }^{2}$ A. V. Zabrodin, Teor. Mat. Fiz. 113, 179 (1997); [ Theor. Math. Phys. 113, 1347 (1997)].

${ }^{3}$ R. 1. Yamilov, J. Phys. A 39, R541 (2006).

${ }^{4}$ F. W. Nijhoff and H. W. Capel, Acta Appl. Math. 39, 133 (1995).

${ }^{5}$ B. Grammaticos, G. Karra, V. Papageorgiou, and A. Ramani, Integrability of Discrete-Time Systems, Chaotic Dynamics, NATO Advanced Institute, Series B: Physics Vol. 298 (Plenum, New York, 1992), pp. 75-90.

${ }^{6} \mathrm{G}$. Darboux, Leçons sur la théorie générale des surfaces et les applications geometriques du calcul infinitesimal, T.2 (Gautier-Villars, Paris, 1915).

${ }^{7}$ I. M. Anderson and N. Kamran, Duke Math. J. 87, 265 (1997).

${ }^{8}$ A. V. Zhiber and V. V. Sokolov, Usp. Mat. Nauk 56, 63 (2001); [ Russ. Math. Surveys 56, 61 (2001)].

${ }^{9}$ A. B. Shabat and R. I. Yamilov, Exponential Systems of Type I and the Cartan Matrices, preprint ed. (Bashkirian Branch of Academy of Science of the USSR, Ufa, 1981) (in Russian).

${ }^{10}$ A. N. Leznov, V. G. Smirnov, and A. B. Shabat, Teor. Mat. Fiz. 51, 10 (1982). 
${ }^{11}$ A. V. Zhiber and F. Kh. Mukminov, in Problems of Mathematical Physics and Asymptotics of their Solutions, edited by L. A. Kalyakin (Institute of Mathematics, RAN, Ufa, 1991) pp. 13-33.

${ }^{12}$ A. A. Bormisov and F. Kh. Mukminov, Teor. Mat. Fiz. 127, 47 (2001); [ Theor. Math. Phys. 127, 446 (2001)].

${ }^{13}$ V. V. Sokolov and S. Ya. Startsev, Theor. Math. Phys. 155, 802 (2008).

${ }^{14}$ A. V. Zhiber and R. D. Murtazina, Fundam. Prikl. Mat. 12, 65 (2006) (in Russian).

${ }^{15}$ N. Kh. Ibragimov and A. B. Shabat, Funkc. Anal. Priloz. 14, 25 (1980).

${ }^{16}$ A. V. Mikhailov, A. B. Shabat, and R. I. Yamilov, Usp. Mat. Nauk 42, 3 (1987).

${ }^{17}$ R. I. Yamilov and D. Levi, J. Nonlinear Math. Phys. 11, 75 (2004).

${ }^{18}$ M. Gürses and A. Karasu, Phys. Lett. A 251, 247 (1999); e-print arXiv:solv-int/9811013.

${ }^{19}$ M. Gürses, A. Karasu, and R. Turhan, J. Phys. A 34, 5705 (2001); e-print arXiv:nlin.SI/0101031.

${ }^{20}$ S. I. Svinolupov, Phys. Lett. A 135, 32 (1989).

${ }^{21}$ A. V. Zhiber and A. B. Shabat, Dokl. Akad. Nauk SSSR 247, 1103 (1979); [Sov. Phys. Dokl. 24, 607 (1979)].

${ }^{22}$ A. V. Zhiber and A. B. Shabat, Dokl. Akad. Nauk SSSR 277, 29 (1984); [Sov. Math. Dokl. 30, 23 (1984)].

${ }^{23}$ I. T. Habibullin, Symmetry, Integr. Geom.: Methods Appl. 1, 23 (2005); e-print arXiv:nlin.SI/0506027.

${ }^{24}$ I. Habibullin and A. Pekcan, Theor. Math. Phys. 151, 781 (2007); e-print arXiv:nlin/0610074.

${ }^{25}$ I. Habibullin, N. Zheltukhina, and A. Pekcan, Turk. J. Math. 32, 1 (2008); e-print arXiv:nlin/0703065. 